\title{
Ottoman-Turkish Manuscripts in the Islamic Studies Library and Other Libraries of McGill University*
}

\section{by Adam Gacek and Ali Yaycioğlu}

The present paper consists of descriptions of 48 individual Ottoman-Turkish texts as found in 35 volumes preserved in the Islamic Studies Library, the Department of Rare Books and Special Collections and the Osler Library of the History of Medicine. Although small in size, the collections of Ottoman-Turkish mantiscripts at McGill are interesting not only from the point of view of scope (some 15 broad subjects) but also because they provide us with a wealth of paleographical and codicological data, which printed catalogues do not always record.

Le présent article se compose de descriptions de 48 textes écrits en turc-oltoman et recueillis dans 35 volumes préservés à la Bibliothèque d'études islamiques, au Département des livres rares et collections spéciales, et à la Bibliothèque Osler de l'histoire de médecine. Bien que peu nombreuses, los collections des manuscrits turc-ottomans à l'Université McCill sont intéressantes non seulement du point de vue de l'ampleur (une quinzaine de sujets) mais aussi parce qu'elles fournissent une abondance de données paléographiques et codicologiques, qui ne sont pas toujours incluses dans des catalogues imprimés.

"We acknowledge here. with gratitude, the generous financial support of Prof. A. Üner Turgay, Director of the Institute of Islamic Studies, for the research done on this collection. We would also like to thank Prof. Mehmet Kalpaklı and Steve Millier for their editorial assistance.

\section{Introduction.}

$\mathrm{T}$ he collections of Islamic manuscripts at McGill University cover the four major languages: Arabic, Persian, Ottoman Turkish and Urdu. The Arabic collections have now been described in a recently published union catalogue and four articles. ${ }^{1}$

The present paper discloses for the first time the hidden treasures of the Ottoman manuscripts which are housed in the Islamic Studies Library (29 vols.), the Department of Rare Books and Special Collections ( 4 vols.) and the Osler Library of the History of Medicine (2 vols.). This small collection consists of 48 main titles. Most of the manuscripts in the Islamic Studies Library were acquired on the recommendation of Niyazi Berkes, a former professor of the Institute of Islamic Studies,
McGill University, from Thornton's of Oxford in October of 1963.

Although small in size, this collection covers a remarkably wide range of subjects. Best represented is sufism with 15 texts (nos, 3, 19, $21,25,27,31,33-36,39,44-47)$. It is followed by ethics (nos. 1, 10, 16, 26, 28, 37, 43), Islamic law, includirg shari a manuals, fetvá collections and legal practices (nos. 4, 5-6, 11-12, 41), poetry (nos. 8-9, 14, 40), medicine (nos. 22-24, 32), and ibādāt (nos. 17, 29). The other subjects represented are: codes of law (Kănūnnäme) (no. 18), Ottoman bureaucracy and statecraft (no. 38), recitation of the Qur'ān (no. 20), fütürvetnāme (no. 13), calendar (no. 2), history (no. 48), late Ottoman military organization (no. 7), calligraphy (no. 15), 
interpretations of dreams (no, 42) and an encyclopedia of various disciplines (no. 30).

The collection contains a complete and elegantly calligraphed, Arabic text of the famous poem Bad' al-amāi (no. 26). In addition, eleven compositions (nos. 3, 17, 19, $26,36-37,40-41,43-45)$ are translations and/or commentaries, the original texts being either in Arabic (nine texts) or Persian (two texts). (of particular interest is an apparently unknown translation of the well-known Persian sufi work Tazkirat al-awliyā' (Tezkiret ül-evliyā'), no. 45 ,

From a paleographical standpoint, the collection also features a variety of scribal hands. The predominant script is neshi (nos. 1 , $3,15,17,19,21,22,26,27,29,32,36,41,43$, 44), neshī with elements of Ottoman ta'lik (nos. $6,12,24,25,45$ ), icảzet (nos. 12 c.II, 18) and rik'a (including rik'a. kırması) (nos. 7-8, 30, 4648). Of special interest here are: (i) $A h k \bar{a} m-I$ $s a \bar{l}$ (no. 2) calligraphed in three scripts: neshī, sülüs and tevkī', (ii) an exquisite copy of

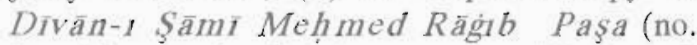
9) calligraphed in Persian nasta' $l i ̄ q$, (iii) nos. 12 c. II and 1.9 with colophons in icāzet, and (iv) an ownership statement (no, 5) in siyākat script.

Most of the colophons and ownership statements are in Arabic. The transcription statements include, on the one hand such expressions as harrarahu or tahrir, sawwadahu or taswid and katabahu or kitābah, and on the other, "an yad and min yad, as opposed to the more usual 'alá yad (in the hand of). Among the expressions introducing statements of ownership are șāhibuhu, istașhabahu, tamallakahu, min mumallakāt, qad tamallaka, dakhala fì milk and intaqala bi-yad.

Among these: statements there are three important attestation/reading notes (imdā') to be found in no. (3. The use of chronosticons for the date of composition is attested in nos 14,19 , 21, 29 and 44. In terms of provenance we find the following towns and/or villages indicated: Kalíbūlī, i.e. Gelibolu (no. 5), al-Urmiyah (no. 11), Țirnuvá (no. 14), al-Qusțanținiyah (no. 21), Tubkhānah, i.e. Tophāne (no. 41) and Qarahyaqah, i.e. Karayaka (no. 45). There are 20 dated texts, of which the oldest is a copy of Minhāc ül-Ísläm transcribed in $906 / 1559$. Three other early manuscripts deserve to be mentioned here. These are Gencīne-i rāZ (no. 14) copied in 1002/1593, 'Imād ül-İslàm (no. 17) copied in 1069/1659 and Biżă'at ülḥ̈̈kkām fì ị̣kām il-aḥkām (no.5), which apparently was copied in 1080/1669-70, i.e., twenty years before the death of its author Hāibzäde: Muhammed bin Muștafá bin Maḥmūd el-İstanbūī. Among the other dated manuscripts, six were transcribed in the $12 / 18$ th and nine in the 13/19th centuries. One (no. 48) was copied as late as 1929 .

All manuscripts were copied on European or European style laid. or wove paper. Wove paper was used in nos. $8,9,21,46,47,48$; no. 9 in particular carries an imitation watermark: BATH. A number of papers were tinted (coloured) (see e.g. nos. 9, 19, 26, 27, 4.4). The laid papers, were usually well-glazed and feature a wide assortment of well-known watermarks. These are:

- anchor in a circle (nos. 14, 20, 29)

- crown-star-crescent (crown surmounted by a star and a crescent) (nos. 6, 17, 18, 20, 32)

- crown and grapes, i.e. bunch of grapes surmounted by a crown (nos. 2, 11, 12c. II, $15,41,44)$

- diadem (no. 11)

- $\quad$ eagle displayed (nos, 1, 19, 36, 41, 44, 47)

- eagle displayed, in a coat of arms (no. 27)

- lion passant guardant (nos. 3, 12c I, I9, 26)

- lion passant guardant, in a coat of arms (nos. 1, 31)

- lion rampant, in a coat of arms (nos. 12c. I, 25, 31, 36, 44, 47)

- paschal lamb (no. 43)

- PICARDO; BP (no. 30)

- scissors (no. 29)

- six-pointed star (no. 11)

- three crescents (nos. 1, 45)

- three crescents with human profiles (nos. 1, 22)

- three hats (nos. 1, 4, 11, 17, 19, 25, 29, 45).

As far as illumination is concerned most of the manuscripts in this collection. were rubricated and gold used for thick rule-borders and headpieces. The use of multiple coloured inks in the text is beautifully illustrated in 
Ahkām-l sāl (no. 2). Thirteen headpieces can also be found in the collection (nos. 1, 3, 9, 12c. II, 25-27, 41, 43, 44). There are four exquisitely executed headpieces in Divān-i Şāmi Meḥmed Rāğb Paşa (no. 9).

The traditional envelope flap (mikleb) in bookbinding was used in only 16 cases. Six bookcovers have no flap (nos. 5, 9, 30, 31, 36, 44) while the Western influence is even more visible in nos. 17, 20,47 and 48. It is interesting to note that in ten cases paper was used as a covering for the bookcovers and that six volumes were covered with marbled paper (ebru). For the more elegant bindings red or brown leather was used. This was usually blindor gold-stamped, the typical decoration consisting of centre-medallions with pendants and border decoration. The best examples here are nos. $2,9,25,27,44$. In one case (no, 41) the center-piece was onlaid and in another (no. 3) velvet was used for the central panels.

The transliteration employed here is for the most part the romanization scheme for Ottoman- Turkish developed by E. Birnbaum², although we have been forced to use the Arabic transliteration scheme of the Library of Congress for names and phrases written in an exclusively Arabic grammatical context. This is the case especially in colophons and most of the ownership statements. For names of Turkish origin but used in an Arabic context, we have provided the Turkish form in parentheses following the Arabic version, e.g., Tubkhānah (Tophane). Furthermore, short incipits have been supplied for the items, which differ and/or could not be found in any catalogues.

\section{Abbreviations}

AMM

BM

$\mathrm{BN}$

$\mathrm{EHB}$

Özege, M. Seyfettin. Eski harflerle hasılmış Türkçe eserler kataloğu. Istanbul, 1971-9. 5 vols.

EI

The Encyclopaedia of

Islam. 2nd edition.

Leiden, 1960-

GAL Brockelmann, Carl.

Geschichte der

Arabischen Litteratur

Leiden, 1943-1949. 2

vols; Supplement,
Leiden, 1937-1942. 3

vols.

(jSO)

Babinger, F. Die

Geschichtsschreiber der

Osmanen und ihre

Werke. Leipzig, 1927.

$\mathrm{HC}$

Yahya, Osman. Histoire et classification de l'oeuvre d'Ibn 'Arabi; étude critique. Damas, 1964. 2 vols.

IA

ISL

Islarn Ansiklopedisi. Istanbul, 1950-86.

Islamic Studies Library.

McGill University.

Osler Library of the

History of Medicine.

McGill University.

OM

Mehmed Tāhir, Bursalı.

'OS manl Mii' elififferi.

Istanbul, 1333/1915. 3

vols. 
PFA

NE:

RBD

SF

TA.

TGK

$\mathrm{TH}(2)$
Ritter, Hellmut. "Philologika

XIV. Fariduddin "At $t$ är

II". Oriens, 11 (1958): 70-76.

Nev'ì Efendi. Netāyic ế-fünün (llimlerin özü), ed. Ömer

Tolgay. Istanbul, 1995.

Department of Rare Books and Special Collections. McCill University.

Şem 'dânî-zâde Fındıklı Süleyman Efendi. Şem 'dânîzâde Findıklı Süleyman Efendi Târihi. Mür'it't-Tevârih, ed.

Münir Aktepe. Istanbul, 1976.

Türk Ansiklopedisi. Ankara, 1948-1984.

Kut, Günay. Terciman Gazetesi Küt üphanesi Tiükçe Yazmalar Kataloğu. Istanbul, 1989.

TH(1) Flemming, Barbara. Türkische Handschriften, Teil

1. Wiesbaden, 1968.

Götz, Manfred. Türkisclie

Handschriften, Teil 2.

Wiesbaden, 1968.
YE

$\mathrm{TH}(3)$

$\mathrm{TH}(4)$

$\mathrm{TH}(5)$

THB

THR

TSM

VH

The Manuscripts
Sohrweide, Hanna. Türkische Hand'schriften, Teil 3.

Wiesbaden, 1974.

Götz, Manfred. Türkische Hand'schriften, Teil 4.Wiesbaden, 1979.

Sohrweide, Hanna. Türkische Handschriften, Teil 5. Wiesbaden, 1981.

Pertsch, Wilhelm. Verzeichniss der türkischen Handschriften der Königlichen Bibliothek zu Berlin. Berlin, 1889.

Rado, Sevket. Türk hattatlart. Istanbul, 1983.

Karatay, F. E. Topkap!

Sarayı Müzesi

Kütüphanesi Türkçe

Yazmalar Kataloğu.

Istanbul, 1.961. 2 vols.

Tschudi, Rudolf. Das Vilâjetnâme des Hâdschim Sultan: cine türkische Heiligenlegende, Berlin, 191.4.

Yunus EInre Divânı, ed. Burhan Toprak. Istanbul', 1960.
1 (ISL 57)

\section{$\bar{A} d \bar{a} b$ ül-menāzil}

Author: ['Abdüllatif bin Durmuş Fakih bin Selim Zeki Karamani] (fi. $2^{\text {nd }}$ half of $10 / 16^{\text {th }}$ cent.). - Composed in $985 / 1577$ (f. 2a). Compendium on ethical and moral issues related to women and family life.

ff. [8] 271; $220 \times 165 / 170 \times 108$ mm; 23 lin. Laid European paper; watemarks: 1. eagle displayed, 2. coat of arms (lion passant guardant), 3. Three crescents with human profiles, 4. three crescents, 5. three hats. Elegant neshĩ hand. - Simple golden headpiece.Double-line rule-borders. - Catchwords. - Text. rubricated (keywords, quotations and omissions in the margins). - Overlinings. - Paper covered boards with flap (flap detached).

Anonymous copy, transcribed in Cemazi elevvel 1233/1817. - Table of contents (ff. [2-5]).Former owner: Sayyid Sayf Allāh (f. la).

Ref.: TH(2), 176; OM, I, p. 130. 
2 (RBD 1)

\section{[Aḥkăm-l săl]}

Calendar for the year Rabí ${ }^{6}$ I $1129 / 1716$ to Rabí I 1130/1718, dedicated to Sultan Ahmed III (reg. 1115/1703-1143/1730).

ff. [10]; $290 \times 185 / 210 \times 130$ mm.; lines vary; text in columns - First folio blank (text begins on f. 2b). - Laid European paper; watermark: crown and grapes. - Executed in elegant neshi, sülüs and tevkí scripts (Fig. 3).- (Golden ruleborders. - No catchwords. - Polychrome text. Golden vegetal designs (ff. 9b-10a). - Goldstamped light brown leather binding; with flap.

Anonymous and undated copy (ca. 1129/1716). Bookplates: Henry B. H. Beaufoy F. R. S. and Presented to the Redpath Library Mccill University from the Alice E. Redpath Fund.

\section{3 (ISL 140)}

\section{Behcet ül-esrātr ve lem at ül-envãr}

Anonymous translation of Umun al-barähinn, also known as Maraàqib al-Shaykh Ahmad al-Rifă 'T, hagiography of the founder of the sufi order, alRifă'îyah, Alumad al-Rifãì (d. 578/1182) (see GAL, SI, 781; EI, VIII, 524b).

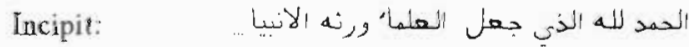

ff. [121]; $225 \times 165 / 140 \times 80 \mathrm{~mm}$; 13 lin. Buff, tinted and glazed laid European paper; watermark: lion passant guardant, FP; VG. Clear neshī hand. - Crudely illuminated doublepage frontispiece. - Borders and discs filled in copper-based ink- Catchwords.- Text rubricated. - Red leather binding with velvet center panels and flap.

Anonymous and undated copy $\left(13 / 19^{\text {th }}\right)$. - Clean copy. - Title with Seal of Solomon above besmele. - Acquisition note, dated 1282/1865-6, on fly-leaf (purchased for the sum of 30 gurus).

$$
4 \text { (ISL 104) }
$$

\section{Behcet ül-fetầá}

Author: [Yeñişehirli 'Abdulläh Efendi] (d. 1156/1743)
Incipit:

$$
\text { يار مسى اولوب معذور اولان زيد يار مسنى... }
$$

ff. [3] $346[+1] ; 255 \times 140 / 185 \times 70 \mathrm{~mm}$; 23 lin. - Laid European paper; watermark: three hats. - Elegant Ottoman ta'lị hand. - Singleline rule-borders in red. - Catchwords. - Text rubricated (el-cevāb, titles). - Blind-stamped brown leather binding with flap.

Anonymous and undated copy (late $12 / 18^{\text {th }}$ cent.). - Table of contents (ff.[Ib-3a]). Marginal glosses in different hands. - Additional fetvás in a different hand (probably that of a former owner) taken from various textual sources and different muftīs. - Two seals: 1. Muh ammed Hüsāmeddīn (f. [3a]), 2. Halìl Hilmì (f. [ $[\mathrm{b}]$ ). - Inaccurate identification of the book by a second hand as Fetãvá-yı Behcet Efendi (f. [la]).

Ref:: $\mathrm{TH}(5), 112$ (different incipit); OM, I, p. 363; EHB, I, p. 122.

$$
5 \text { (ISL 147) }
$$

\section{Bizà̃at ül-ḥükkām fi ị̣kām il-ahkām}

Author: Häcibzāde Muhammed bin Mustafá bin Maḥmūd el-İstanbūlī (d. 1100/1688-9). - Treatise on Ottoman legal practice including sample legal decisions issued by shari'a courts, compiled between 1.064/16534 and 1081/1670-1 (!).

ff. [4] $157 ; 210 \times 165 / 170 \times 100 \mathrm{~mm}$; 25 lin. Laid European paper; watermark: unidentified. Single-line rule-borders. - Neat Ottoman ta'lik hand.- Text rubricated.- Blind stamped; brown leather binding; no flap.

Copied in Kalībūī (Gallipoli or Gelibolu) by (tahrīr "an yad) Hasan Sidqi, secretary at the court (mahkamah) of Gallipoli, 1080/1669-70. Table of contents. - Omissions in the margins. Ownership statements and two seals (f. [1 a]) by: 1. (bādalat) Muhammad ibn Khalìl, 2. (min mumallakāt) Ạ̣mad Nazị̂ ibn Husayn ibn Muhammad ibn Aḥmad al-Samāqawīi, 3. (săhịib) İbrähưm bin Mollā 'Ali...(?), dated 1211/1796-7 (siyak āt script). - Inserted in an envelope: an illuminated calendar for Ramadān (wove paper, $750 \times 75 \mathrm{~mm}$.).

Ref.: TSM, I, 318; OM I, p. 280. 
6 (ISL, 154)

Defter-i sukük

Author: [Mehmed bin Derviş Mehmed], known as Șāni-i Edirnevĩ (d. 1033/1624).Manual of sample legal decisions issued by shari'a courts, composed in 1033/1623-4.

ff. $39 ; 260 \times 145 / 150 \times 80 \mathrm{~mm} ; 15$ lin. - Laid European paper; watermark: crown-starcrescent; trefoil PA. - Neshī/ Ottoman ta'lik hand. - Catchwords. -Text rubricated. - No binding.

Anonymous and undated copy (copied possibly ca. Cemāzi el-evvel 1109/1697, see no. 3 below). - Incomplete text (bāb 1-4 only). - Some marginal glosses. - Three ownership statements and two seals, one in the shape of a lion with a halo (f. la) by: 1. (tamallakahu) 'Alī' Qabazādah al-Qayșarawi, 2. 'Âli bin eş-Şeyh 'Ömer, 3. note on the birthday of Matlabacı Mahmund (elebin's daughter (13 Cemāzi el-evvel 1109/1697). Three attestation / reading statements (imḍā')

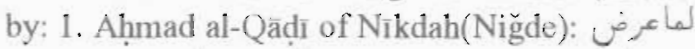

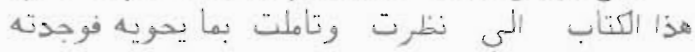

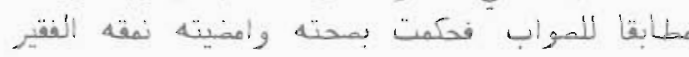

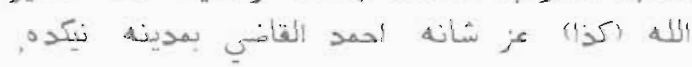

2. "Alī ibn 'Úmar al-Mudarris, 3. 'Alī alMawāì̄. - Some verses and notes (f. 39b).

Ref: TGK, I, 57.

$$
7 \text { (ISL 171) }
$$

Devlet-i 'aliyye-yi 'Oșmāniye 'niñ tensikāt-1 askerīyesi

Anonymous compilation of writings on history of Ottoman military and copies of some legal documents on late $19^{\text {th }}$ or early $20^{\text {th }}$ cent. Ottoman military organization.

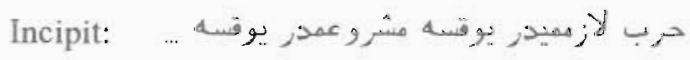

ff. $[474] ; 194 \times 130 / 170 \times 110 \mathrm{~mm}$; 13 lin. Laid paper; no watermark. - Ottoman rik'a hand. - Text in black ink. - No catchwords, - Black quarter-leather binding with center stamp of Ottoman dynasty; no flap.

Anonymous and undated copy (early $20^{\text {th }}$ century).
8 (ISL 174)

\section{Dĩvān-I Kaygususuz Sulțān}

Author: Kayg usuz [Abdāl] (fl. $9 / 15^{\text {th }}$ cent.).

ff. [85]; 285 x $195 / 220$ x 135 mm.; lines vary.Wove and. laid paper. - Riḳ'a ḳrması hand. Text in columns and compartments. - Some catchwords missing.- Some lacunae (ff. 79, 8183).- Double-line, dark-red rule-borders. Modern green cloth binding.

Anonymous and undated copy (late $13^{\text {th/ }}$ / early $20^{\text {th }}$ cent.), - Some corrections in pencil.

Ref:: $\mathrm{TH}(1), 424$.

$$
9 \text { (RBD 2) }
$$

\section{Dîān-J Şāmi Meḥmed Răğ}

Author: Rāgıb Paşa, known as Hoca Mehmed, or Koca Rảgıb Paşa (d. 1176/1763).

Incipit:

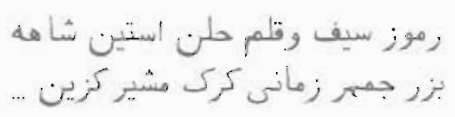

ff. [128]; $205 \times 125 / 135 \times 80$ mm.; 15 lin. Colored wove European paper (light blue, pink and yellow); imitation watermark: BATH (f, 2).Calligraphic Persian nasta'līq hand. - Four exquisitely illuminated headpieces (ff. $1 \mathrm{~b}, 6 \mathrm{~b}$, 16b, 18b) (Fig. 1). - Golden rule-borders. Catchwords. - Chapter headings in blue and red.Blind and gold-stamped red leather binding; no flap.

Copied (taswī') in Dhu al-Hijjah 1260/ 1844 by Muḥammad "Alī ibn Muḥammad Ṣādiq alShīrāzi.

Ref:: TH(3), 247 (different incipit); EI, VIII, pp. 390-391; EHB, I, p. 293.

$$
10 \text { (ISL 50) }
$$

\section{Fażīletnāme}

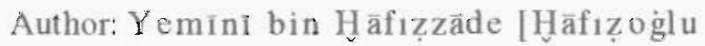
Derviş Mehmed, known as (mahlaș) Yemint] (fl. $1^{\text {st }}$ half of $10 / 16^{\text {th }}$ cent.).- Mesnevi 
extolling the virtues (fażilet) of 'Alr and his family.

ff. [24a-256a] 1-235 (original foliation); (for other codicological and paleographical details see no. 31).

Anonymous and undated copy (ca, $1263 / 1846-$ 7; see no. 34), - Table of contents (ff. 22b-23b).

Ref: TH(2), 254-6; EHB, I, p. 386.

\section{1 (ISL 11)}

[Fetävá-yI "Alî Efendi]

Author: [Çatalcalı 'Alī Efendi] (d. 1103/1692).

ff. [6] $336 ; 305 \times 165 / 185 \times 65$ mm.; 31 lin. Laid European paper; watermarks: 1. six-pointed star GV (f. [1]), 2. three hats, 3. crown and grapes, 4. diadem GB. - Neat Ottoman ta 'lik hand (Fig. 8), -Single-line rule-border with outer frame in red. - Text rubricated (el-cevāb and subtitles). - Catchwords. - Marbled paper covered boards, with flap.

Copied by (sawwadahu) Sulaymān ibn Hamzah in 1189/1775-6 in al-Urmiyah.- Clean copy.Table of contents (ff, [1 b-3a]).- Ownership statement on f. [1a]: (tamallakahu) Ḥâfiz: Ibrāhīm, dated 1219/1804-5.

Ref.: TH(3), 110; TH(4), 79; EHB, I, p. 402.

\section{2 (ISL 56 and 169)}

\section{Fetāvá-yI 'Ațā'ullāh Efendi}

Author: [Minḳārīzāde Yahyyá Efendi] (d. 1088/1677). - Compiler: [Meḥmed] "Aṭâ."ullāh Efendi (d. 1127/1715).

Copy I (ISL 56): Title on tail. - ff. [5] $396[+4$. blank] ; $240 \times 170 / 180 \times 105$ mm.; 27 lin. Laid European paper; watermarks: 1. coat of arms (lion rampant), 2. lion passant guardant. Neat neshī / Ottoman ta'lik hand. - Text rubricated. (el-cevāb, titles, overlinings). Catchwords. - Blind-stamped, brown leather binding with flap.

Copied by ("an yad) Aḷmad ibn "Umar; no date $\left(12 / 18^{\text {th }}\right.$ cent.).- Table of contents (f. [4b-5a]). -
Marginal glosses and fetvás by, among others, 'Alì, müfti at Kesrìye (Kastoria); müftĩ of Sis; Muștafá, müftĩ at Ưsküb (Skoplje); 'Abdullāh, müftī at Ergirikasri (Gjinokastër) ; 'Abdüșșamed, müftì at Yanya (Ioannina); 'Atạ'ullāh, müfti at: Yanya; Halīl, müftri at Holumiç (Chlomoutsi); Muhammed, müftī at Kesrìye; 'Alī, müftì at Kula، - f. [398b] contains Tercihh-i beyinat taken from al-Muhịt alBurhānī. - Inserted in an envelope: an original fetvá signed by Ahmed, Müftī...?

Copy II (ISL 169): ff. [4] 280; 270 x 160/210 x $110 \mathrm{~mm}$; 33 lin. - Laid European paper; watermark: crown and grapes. - Clear neshi hand with many elements of icāzet. - Simple golden headpiece; golden single-line rule-borders (f. $1 \mathrm{~b}$ 2a, 241a, 250b, 251a, 260b). - Catchwords. Polychrome text (mes'ele in blue, el-cevāb, chapter-headings, subtitles and overlinings in red). = Some side-heads trimmed.- Modern blue cloth binding, no flap.

Copied by (tahrir; min yad) Darwish Muṣṭáá ibn Muḥammad (colophon in icāzet hand, Fig. 5) in 1189/1775-6. - Table of contents (f. [3b-4b]). - Marginal corrections. Ownership statement (tamallakahu) (f. 1a): Aḥmad Rushdi ibn Muhammad Nūrī, known as Aṭah (?) Nābīzādah.

Ref: $\mathrm{TH}(4), 80$.

\section{3 (ISL 128)}

\section{Fütüvvet üss-şeriff}

Anonymous fütüvvetnāme.

Incipit:

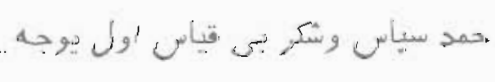

ff. $[47 \mathrm{~b}-51 \mathrm{~b}]$; (for other codicological and paleographical details see no. 36 ).

No colophon ( copied ca. 1267/1850-1, see f. 61a).

$$
14 \text { (ISL, 75) }
$$

\section{Gencīne-ì rãz}

Author: Yaḥyá [Beg Dukākīnzāde] (fl. $1.0 / 16^{\text {th }}$ cent.). - Collection of verses of ethical 
and didactic nature. - Composed in 947/1540-41 (see chronosticon: hูāmūs, on f. 85a).

ff. [1b-85a]; $195 \times 125 / 150 \times 75$ mm.; 17 lin.Laid European paper; watermarks: 1. anchor in circle; trefoil ZM, 2. unidentified. - Clear Ottoman ta'līk hand; (ff. 51-54 supplied by a different hand, neshī).- Single-line rule-borders in red; text in columns. - Catchwords. - Text rubricated. - Blind-stamped brown leather binding with flap.

Copied by (katabahu) Alhmad ibn Shaykh Bayrām in Țirnuwá (Ṭirnova) in 1002/1593. Some omissions in margins.- Four ownership statements with three seals (f. la): 1 . (istașḩ̣abahu) Muștafá Dukkānī, dated (seal) 1180/1766-67, 2. (qad tamalakka) Husayn alDukkānī, 3. (dakhala fi milk) Muhammad Shākir, secretary (kătib) in the Imperial Court (Divān-1 Humāyūn), 4. (intaqala bi-yad) Muhammad Țāhir al-Mawlāwi .

Ref: $\mathrm{TH}(2), 210-211$.

\section{5 (RBD 3)}

[Gülzär-l sevăb]

Author: [l̉brăhim bin Muștafá], known as Nefeszăde (d. 1060/1650). - Treatise on calligraphy, paper and ink. Also known as Tezkiret ül-hyattatin. Titie on f.la: Nefesĩzāde (!) Risālesi.

ff. [30]; $175 \times 110 / 120 \times 70 \mathrm{~mm}$; 11 lin. - Laid European paper; watermark: crown and grapes ...LANGUEDOC. - Clear, partly vocalized neshū hand. - Text rubricated. - Catchwords. - Paper wrapper.

Anonymous copy. - Transcribed (tahrīr) in Rabi ${ }^{*}$ II 1161/1748. - Approximately two first folios wanting.

Ref.: TH(1), 269; TH(4), 523-5; THR, p. 96.

16 (ISL 153)

\section{'Ibretnümānàne}

Anonymous collection of edifying stories (hikāyăt).

Incipit: يدمه عبرت نماناهه خلان مفا واخوان

ff. [30b-47a]; (for other codicological and paleographical details see no. 18).

Anonymous and undated copy (early $12 / 18^{\text {th }}$ cent.). - Main text followed by: 1. Simurg-1 ankā hikāyesi (ff. 47b-49a), 2. two passages from Dürr-i meknün possibly by Ahmed Bicän Yaziciogl (fl. $9^{\text {th }} / 15^{\text {th }}$ ) (ff. 49a-50a) (TH, IV, 333), 3. Gazel-i Sultạn Murād (f. 50b), 4. Münäcät-1 Şeyh Sa'dī, with two seals depicting a lion with a halo (f. $51 \mathrm{~b}$ ).

\section{7 (ISL 138)}

\section{['İmäd üI-İsläm]}

Author: ["Abd al-"Aziz Fārisi]. Translation and commentary by "Abdürrahmōn bin Yũsư el-Aksarāyī (fl. $9 / 15^{\text {th }}$ cent) upon 'Undat al-İslàm, a Persian treatise on the five pillars of Islam ('ibādāt). Title in colophon: "Undat al-İstāun.

ff. [4] 310 (shaved in places) (pp. 675!, supplied later); $195 \times 140 / 115 \times 90$ mm.; 19 lin. - Laid European paper; watermarks: 1 . unidentified coat of arms, 2. three hats, 3. crown-starcrescent; trefoil $A G$. - Neshī hand influenced by other styles. - Catchwords. - Text rubricated. Side-heads (matlab). - European black quarterleather binding.

Copied by (katabahu) Maqșūd ibn Munlā Sayyidī in Jum. I 1069/1659 (f. 305b). Marginal omissions and corrections. - Text preceded by a table of contents (ff. [1b-4]]). f.305b: date of death of 'Ayşe Hâtūn, 1178/1764-5. - f.1 a: prayer, dated 1115/1703-4, Followed by an anonymous short composition on ethics, f. 306a-310b, beginning (after the basmalah and hamdalah): Evvel 'ālem-i faburī...

Ref.: BM, p. 12-13; TSM, I, 235; OM I, p. 265.

$$
18 \text { (ISL 153) }
$$

\section{[Ķanunname]}

Compiler: ['Alı̀ Mü'ezżinzāde, known as 'Aynī 'Alī] (fl. 11/17' cent.). - This work, also known as Kavāniñ-i $\bar{A} l-i$ 'OŞmān der 


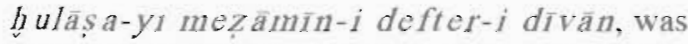
dedicated to Sult an Ahmed I (reg, 1012/16031026/1617) (f.1a).

ff. [1a- 19b]; $205 \times 145 / 160 \times 90$ mm.; 19 lin. Laid European paper: watermark: crown-starcrescent: trefoil BG. - Elegant nesh̆i / icāzet hands. - Catchwords. - Text rubricated. Marginal glosses. - Binding missing.

Anonymous and undated copy (early $12 / 18^{\text {th }}$ cent.). - First leaf missing. - Followed by a description of Istanbul (Kostantinīye) from Aużah ül-mesālik of Mụ̣ammed Sipāhizāde (d. 997/1589, GAL, SII, 44) (f.19b).

Ref.: TH(1), 348; EHB, II, p. 847.

$$
19 \text { (ISL 58) }
$$

Kaşf ül-ḥicāb an vech il-kităb

Author: Yahyá bin "Alī Nev'î (d. 1007/1598-9). - Commentary on Fusus alḩikām (f. la and tail) of Muhyì al-Dìn ibn 'Arabī (d. 638/1240). - Composed in 1002 / 1593-4 (chronosticon: aḩ ar ül-kelām, f. 334a).

ff. [3] 344; $230 \times 160 / 165 \times 100$ mm.; 21 lin. Laid, yellow, tinted and well glazed European paper (except first three folios); watermarks: 1. eagle displayed, 2. lion passant guardant; $\mathrm{AFG}$, 3. three hats. - Elegant neshĩ hand. - Polychrome headpiece; double-line rule-borders.- Text rubricated (qawluhu and overlinings). - Blind and gold-stamped black leather binding, with flap.

Copied by (kitābah, 'an yad) Aḥmad al-Ḥ̣amdi Kümuljunahwī (Gümülcünevĩ) in 1244/1.828-9 (colophon in icāzet hand, Fig. 4), - Table of contents (f. [2b]). - Marginal glosses and corrections.

Ref:: TSM, I, 157-159; GAL, S1, 794.

\section{0 (RBD 4)}

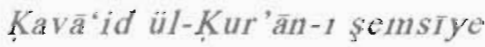

Short, anonymous composition on rules for the recitation (tecvīd) of the Qur'ān. Main part of the text divided into ten chapters (f. 6a) such as: 1. merātib ül-ahkām in-nūn is-sākine, 2.merātib- i medler, 3.merātib-i maksūre... (appears to be different from TSM, I, 7 and THB, 12a).

Incipit: اعلم يتنى بلكل كم قران تجويد ايله..

ff. $21 ; 195 \times 125 / 165 \times 70$ mm.; 27 lin. - Laid European paper: watermarks: 1. crown-starcrescent; trefoil $\mathrm{AG}$; trefoil $\mathrm{bV}, 2$. anchor in circle. - Ottoman ta lịk hand.- Text rubricated.Catchwords.- Marbled paper half-vellum binding.

Anonymous and undated copy (late $11 / 17^{\text {th }}$ or early $18^{\text {ih }}$ cent.). - No preface. - Presented to the Library by Lady Roddick. - Bookplate: In Memoriam Peter Whiteford Redpath B.Sc. Jocelyn Clifford Redpath, B.C.L.

$$
21 \text { (ISL 74) }
$$

\section{Kitāb ün-necāt}

Author: Ismá'il Hakki $\overline{1}$ [Celveti]] elBurusavī (d. 1137/1725). - Handbook for Sufì adepts. - Composed in Dhū al-Hijjjah 1131 1 1.791. (chronosticon: ḩatm-t kelām).

ff. [125]; $230 \times 150 / 160 \times 105$ mm.; 25 lin. Wove European paper; no watermark.- Neat neshī hand.- Single-line rule-borders.Overlinings, in dark-red.- Side-heads.Catchwords. - Plain, red leather binding with flap (marbled paper doublures).

Copied by ('an yad) Ahmmad ibn Ạ̣mad alAnqarawi known as Qarah (Kara) Shaykhzādah, in the reign of Sult an Abdülaziz, 1288/1871, in aí-Qusț anținìyah (Kosțanținiye). - Marginal corrections. - End leaves (thick paper) with a printed statement to the effect that this paper is to be used for wakf purposes.

Ref.: TH(2), 46; EHB, II, p. 905.

$22($ OL $389 / 18)$

\section{$[K i t a \bar{b}-1, t, b b]$}

Anonymous medical treatise containing such chapters as : fă'ida li-ḥaraḳān il-bevl (p. 28); fầ'ida lil-gișāve ve'l-humre (p. 29); șıfăt-1 ma cū n (p. 30); șfăt-1 haubb-1 sultān (p. 30) . 
pp. $28-63 ; 225 \times 160 / 170 \times 100 \mathrm{~mm}$.; 25 lin.. Laid European paper; watermark: three crescents with human profiles. - Clear neshi hand. - Catchwords. - Text rubricated. - Red cloth binding.

Anonymous and undated copy (early $13 / 19^{\text {th }}$ cent.). - Incomplete at beginning and end.Preceded by $M a j m u{ }^{*}[f i a l-t i b b]$ (Arabic) (see AMM, 117).

$23($ OL 389/7)

$[K i t a b-1+1 b b]$

Anonymous medical treatise.

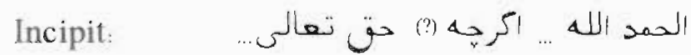

ff. [61b-70b]. - Laid paper. - Ottoman ta'lik hand. - Ink offset. - Water-stained. - Rubrics not supplied. - Catchwords.- For other codicological details see no. 24.

Incomplete (13/19 ${ }^{\text {th }}$ century).- Followed by (f. $71 \mathrm{a}-87 \mathrm{~b}$ ) fragments from various unidentified medical texts.

$$
24 \text { (OL 389/7) }
$$

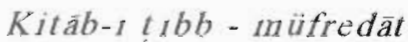

Author: Şāhīn Bey bin. Murād (?). - Book of simple remedies (herbal) arranged alphabetically beginning with bāb-i evvel ìn kamcā (?)

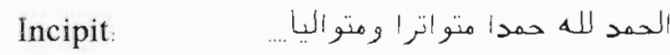

ff. $[1 \mathrm{~b}-32 \mathrm{~b}] ; 200 \times 140 / 180 \times 110-125 \mathrm{~mm}$; 17-21 lin. - Laid European paper; watermark invisible. - Neshi / Ottoman ta'lik hand. Catchwords. - Text rubricated. - Badly waterstained.- Modem style binding with raised bands and title in gold on the spine in Arabic (Mufrad̄at fì al-țibb).

Incomplete at end (probably one or several folios missing, $11 / 17^{\text {th }}$ cent). - Marginal glosses and corrections, some shaved. - Bookplates: 1.Dr. M. Meyerhof (in Arabic), 2. Casey A. Wood. - Main text followed by (ff.33a-58a and 7 la-b) unidentified fragments from a medical treatise divided into fusul from portion of second fașl to thirty seventh fașl (second faṣl: fasl-1 sersām; third: fașl-ı şabbāt; fourth: faṣl-1 seher; fifth: fașl-1 misyān...).

$$
25 \text { (ISL 59) }
$$

Menăkib-1 Seyh Şa bān Veli

Author: "Ömer el-Fu'āī̄ Efendi (d. 1046/1636-7). - Hagiography of Şeyh Sa'bān Veli composed in $1017 / 1608-9$.

ff. $[86,+4$ blanks]; $220 \times 135 / 150 \times 70 \mathrm{~mm}$; 19 lin. - Laid European paper, watermarks: 1 three hats; FFA, 2. coat of arms (lion rampant); (iFA.- Ottoman ta'lik hand with many elements of neshir. - Simple but elegant golden headpiece; single-line red rule-borders.- Catchwords.- Text rubricated (overlinings, keywords, quotations).Dark red leather binding with golden borders and center-pieces (flowering plant), flap missing,

Anonymous copy; dated 1145/1732-3. Followed by (f. 86a-b) a quotation from a work of Muhyī al-Dīn ibn 'Arabī (d. 638/1240).

Ref.: TGK, I, 163-164.

26 (ISL 62)

\section{Menazil ül- 'àrifin}

Author: [Muḥammad al-Ghazzāīi] (d. 505/1111). - Translated extract with a commentary from $K \bar{n} m \bar{i} y a{ }^{\prime}, a l-s a^{\circ} a d a h$ (GAL, I, 540, no. 29) by Şemsediīn es-Sivàisi (d. $1006 / 1597)$.

ff. $[1 b-63 b] ; 235 \times 160 / 175 \times 95$ mm.; 13 lin.Laid, light-brown tinted paper; watermark: lion passant guardant; A.G. - Clear, mostly vocalized neshi hand (Fig. 6). - Simple golden headpiece; rule-borders filled with gold (ff.1b-2a) - Text rubricated (key words, quotations). - Marbled paper covered boards with flap.

Anonymous copy, transcribed probably by Muhammad Țāhir Rūh̄̄, pupil of Mụhammad alWasti, ca. 1240/1824-5 (f. 73b), - Followed by: 1. (f. 64a-66a) Hadith enumerating names of the prophets, 2. (f. 66b-70b) Bahs ül-esmà' il-hüsnà, 3. (f. 71 a-73b) Bad' al-amālī (in Arabic) by Sirāj al-Dīn 'Alī ibn 'Uthmān al-Ūshì (fl.569/1 1.73), see GAL, I, 552; SI, 764. 
Ref: TH(2), 94-95.

\section{7 (ISL 148)}

Meslek üt-tălibinn ve't-'abidin [ve\% văsilfin]

Author: "Abdullāh Simāvì, known as liāhī Efendi $(896 / 1490-1$, or $893 / 1488$, f. 1a). Handbook for sufi novices.

ff. [1] $146 ; 210 \times 130 / 145 \times 75$ mm; 15 lin. Laid, buff tinted European paper; watermark: coat of arms (eagle displayed) GIORO MAGNANI; AL MASSO. - Clear neshi hand. Simple golden headpiece; single-line ruleborders.- Text rubricated (keywords, overlinings). - Brown leather binding with flap and gold-stamped borders.

Anonymous and undated copy (copied before 1283/ 1866-7, f. la).- Ownership note: 'Ali Kamāl ibnn 'Alí al-Murtaḍa iḅn al-Hạjij Husayn, dated 1339/1920-1 (i. 146b).

Ref: $\operatorname{TH}(2), 58-63$.

\section{8 (ISL 75)}

\section{Miftah-1 cennet}

Author: [Ferid Ahmed Bey (Paşa)] (d. 990/15867). - Short anonymous treatise on religious ethics in 8 sections (bāb) without preface.

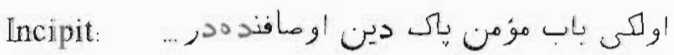

ff. [86a-88b]; (for other codicological and paleographical details see no. 14) .

Anonymous and undated copy (ca. 1002/1593, see no. 14).

Ref.: OM, III, p. 364; EHB, III, p. 1144

\section{9 (ISL 12)}

\section{Minhăc üI- İslām}

Author: Muhammed bin Isma'il (fl. 923/1517).- Manual of religious observances ('ibādāt).- Composed (chronosticon: zuhrr-i kavĩi) in $923 / 1517$ (f. 5a, lin. 11).

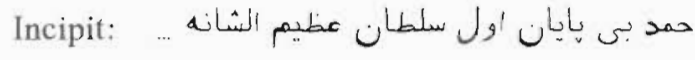

ff. [2] $250 ; 300 \times 170 / 250 \times 130$ mm.; 23 lin. Laid European paper; watermarks: 1. scissors, 2. anchor in circle, 3. three hats; trefoil SS (ff. 150159 supplied later).- Elegant, partly vocalized neshū hand. - Some lines in bold. - Catchwords. Foliation: verso of folios. - Text rubricated. Paper covered boards; flap missing.

Anonymous copy, transcribed (taswīd, 'an yad) in Jumādá I 966/ 1559 (f. 250b). - Marginal glosses. - Text collated (omissions, corrections, etc.).- Fonmer owner (istașhabahu) Dārnădzādah Fayḍ Allāh (f. [2a]), - Invocations: 1. yā kebikec yā hạì (f. [2a]), 2. yā Fettāh yā 'Alìm (above besmele) (f. lb). - f. [1 a] contains a hadith relating to prayer on the authority of Abu Bakr.

Ref.: TGK, I, 73; TH(4), 32 (attributed to 'Abdullāh bin Ismā'îl, different incipit).

$$
30 \text { (ISL 63) }
$$

\section{Netā'ic ïi-fünān ve mehāsīn-i mütūn}

Author: [Yahyá bin 'Alī el-Nev'i]] (d.1007/1598-9).- Encyclopedia of twelve disciplines dedicated to Sultan Murad III (reg. 982/1574-1003/1595).

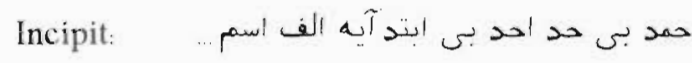

ff. [79]; $265 \times 185 / 220 \times 140 \mathrm{~mm}$; 21 lin. Laid European paper; watermark: PICARDO; BP. - Legible rik" a kurması hand. - Single-line rule-borders. - Catchwords. - Text heavily rubricated (chapter headings, overlinings, diagrams, etc.). - Marbled paper covered boards; no flap.

Anonymous copy, transcribed in Shawwāl 1247/1832. (f. 77b). - Some marginal glosses. Side-heads. - Main text followed by magical formulae (f. 78a). - Inserted in an envelope: six short prayers on tiny scraps of paper and a letter informing the local meclis of Kalburca of the arrival of a preacher (wā' 'iz) dated 1303/1885-6, with the seal of Hasan Seză'y in Turkish.

Ref: TH(1), 372; NE. 


$$
31 \text { (ISL 50) }
$$

Pendnäme, also known as Delil-i budalāa

Author: Kaygusuz [A bdāl] (fl. $9 / 15^{\text {th }}$ cent.). - Sufi treatise containing edifying stories.

ff. $\quad[1 \mathrm{~b}-22 \mathrm{a}] ; 240 \times 175 / 190 \times 120 \mathrm{~mm}$; 17 lin.- Laid European paper; watermarks: 1. lion passant guardant, 2. coat of arms (lion rampant).- Neshī / Ottoman rik'a hand. Catchwords. - Text rubricated. - Leather and marbled- paper- covered boards; no flap.

Anonymous and undated copy (ca. 1263/18456, see no. 34). - Donation statement: gift from Hüseyin Baba of Birecik who was post-nişin of Bektaşi Hูānkāhı to Hasan Hulkị Riżā. - Inserted in an envelope: 'Arsh-i a lá, cosmological chart $(520 \times 375 \mathrm{~mm}$.), written on laid European paper; watermark: crescent with human profile.

Ref: $\operatorname{TH}(2), 86$; TH(3), 84.

$$
32(\text { OL } 389 / 7)
$$

Risāle-i manzūne fì illm-i tibb

Author: most probably Nidā' I (d. after 975/1567-8), - Medical didactic poem.Composed in the time of Sultan Selim II (reg. 974/1566-982/1574).

Incipit:

$$
\begin{aligned}
& \text { الهى حكمت اسمى هحتشم سن }
\end{aligned}
$$

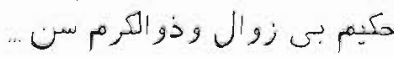

ff, [88a-141b]. - Watermark: crown-starcrescent. - Clear neshi hand. - Rubrication. Badly water-stained.- For other codicological details see no, 24 .

Anonymous and undated copy (early $12 / 18^{\text {th }}$ cent).

Ref: TH(3), 203; TH(4), 400; TH(5), 186 (same explicits but different incipits).

$$
33 \text { (ISL 128) }
$$

[Risăle-i must talahăt-i șüfye]

Anonymous treatise on sufi discourse.

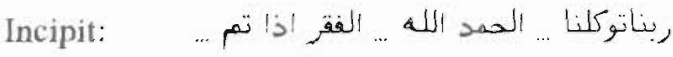

ff. [41a-44b]; (for other codicological and paleographical details see no. 36).

No colophon. - Copied ca. 1267/1850-1 (f. 61a).

$$
34 \text { (ISL 50) }
$$

[Risāle-i ṣufiye]

Anonymous treatise in the form of a conversation between a pupil and his master.

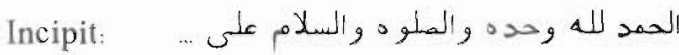

ff. [236b-344b]; (for other codicological and paleographical details see no. 31 ).

Copied (katabahu) in 1263/1845-6 by Șādiq, follower of (haq-pāy-i) Naqshaband Muhammad Bahà' al-Dīn.

35 (ISL 128)

Risāle-i şerîf

Anonymous sufi commentary on a hadith: $\quad ن$

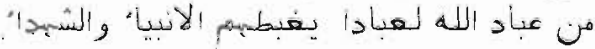

Incipit:

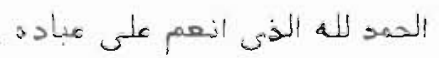

ff. [44b-47b]; (for other codicological and paleographical details see no. 36 ).

No colophon. - Copied ca. 1267/1850-1 (1. 61a).

$$
36(1 S L, 128)
$$

Risāle-i vahtet ül-vucūd

Author: [Muhyi al-Din Muhammad ibn 'Arabil (d. 638/1240). - Translation of Risälah fi al-ahadiyah, also known as Risălat alahadiyah, Risālat al-wujudìyah and Risāiah fi sharh tna'ná qawl al-nabi: Inan "arafa natsalt faqad 'arafa rabbah, possibly by Hüseyin Hüsnü bin Ismài îl.

Incipit:

ff. [1b-14b]; $240 \times 170 / 180 \times 120 \mathrm{~mm} . ; 15$ lin.Laid European paper; watermarks: 1. eagle 
displayed, 2. coat of arms (lion rampant). - Clear neshri hand. - Catchwords. - Overlinings.Marbled-paper-covered boards; no flap.

No colophon. - Copied ca. 1267/1850-1. (f. 61a).

Ref.: HC, I, 13; II, 621; GAL, I, 579 (no. 104); SI, 798 (no, 108).

$$
37 \text { (ISL, 128) }
$$

Risāle-i vālideyn - te’dīb ülmütemerridin

Author: $\mid$ Awh ad al-Dīn 'Abd al-Ahad alNūrì] (d. 1061/1651). - Anonymous translation of the author's Ta'dib al-mutamarridin fi haqq al-abawayn.

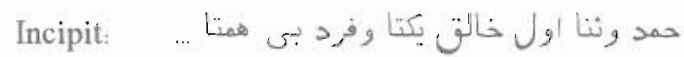

ff. [15b-33b]; (for other codicological and paleographical details see no. 36).

No colophon. - Copied ca. 1267/1850-1 (f. 61a).

Ref:: OM, I, p. 121; GAL, II, 591; SII, 662.

$$
38 \text { (ISL 153) }
$$

Risăle-i vazịfe-h̆̄ărān merātib-i bendegăn-i $\bar{A} l-i \cdot O \operatorname{sinān}$

Author: "Alī Mü'ezzinzāde, known as 'Ayn-i "Alr. - Treatise on Ottoman bureaucracy and statecraft.

ff. [20a-30a]; (for other codicological and paleographical details see no, 18),

Anonymous and undated copy (early $12 / 18^{\text {th }}$ cent,).

Ref:: GSO, p. 141; EHB, IV, p. 1482; BN, 128, $885,1092$.

$$
39 \text { (ISL 128) }
$$

[Su'àl ve cevāb]

Anonymous questions and answers relating to mysticism.

Incipit: الحمد لله الذ لم يتخذولدا وما توفيقى... ff. [34a-41a]; (for other codicological and paleographical details see no. 36 ).

No colophon. - Copied ca. 1267/1850-1 (f. 61 a).

$$
40 \text { (ISL 128) }
$$

[Şerhli-i ebyāt-1 Yünus Emre]

Anonymous commentary on nine verses from the Divān of Yūnus Emre (1l. $7 / 14^{\text {th }}$ cent.). Composed in 1117/1705-6.

ff. [52a-61a]; (for other codicological and paleographical details see no. 36).

Dated $1267 /$ 1850-1. - Followed by 29 verses from Dĩvān of Seyyid 'İmādüddīn Nesīmī (d. 821/1418, see İA, IX).

Ref:: YE, p. 209-222.

$$
41 \text { (ISL 132) }
$$

Şerḥ-i Mültaḳál-ebḥur

Author: [Muḥammed Mevkúfātī] (d. 1065/1654). - Translation of and commentary on Multaqá al-abhur, an Arabic manual of Hianafit law by Ibrāhīm al-Halabĩ (d. 956/1549) (GAL, II, 432; GAL, S II, 642), - Identified by a later hand (dated Safar 1291/1874) as Mültaká tercemesi by Hamdi Efendi (see fly-leaf and tail).

ff. [3] 686; $205 \times 150 / 160 \times 100$ mm.; 21 lin. Glazed laid European paper; watermarks: 1. crown and grapes, 2. eagle displayed. - Clear neshyi hand. - Some quires (10 folios each) numbered. - Simple golden headpiece; doubleline rule-borders. -Irregular catchwords. - Text rubricated (overlinings). - Water-stained (ff. 110) and wormed. - Dark-brown leather binding with flap; gold-stamped borders and onlaid center-pieces.

Copied (harrarahu) in Dhū al-Hijjah 1188/ 1775 by Ismā'il, Shaykh of the reciters (alqurrā') at the mosque of Tubkhānah (Țophāne), Istanbul and a pupil of Abu Muhammad "Abd Allāh ibn Muhammad, known as Yuusuf Afandizādah (Efendizāde) (f. 686a). - Table of contents (ff. 1-3). - Clean copy. 
Ref.: TH(4), 66; EHB, III, p. 1261.

$$
42 \text { (ISL, 75) }
$$

\section{Ta'birr-i düiş}

Short anonymous treatise containing 28 rules for the interpretation of dreams.

Incipit: .بو دوش تعبيراتى مظلمبم مختصر فايدهلو...

ff. [89a-b]; (for other codicological and paleographical details see no. 14).

Anonymous and undated copy (ca. 1002/1593, see no. 14). - Followed by: 1. ff. $90 \mathrm{~b}-91$ b short text containing two dẩires by Tatar Îbrāhīm Efendi on cosmology, 2. ff. 92a-96a various verses, some by Bākī Efendi, 3. ff. 97b-99b story from the life of Prophet Muhammad ending with Du 'â' 'ul-istighfar and a number of verses.

$$
43 \text { (ISL 141) }
$$

\section{Terceme-i nis āb ül-iht tisāb}

Author: Sirajj al-Din 'Umar ibn Muhammad ibn 'Awḍ al-Sha'mī alSunnāmi (fl. 993/1585). - Translation and commentary by 'Alī Cevherī Efendi, known as 'Alì bin Balī (d. 991/1583), on Nisāb al-ịhtisāb (GAL, II, 403; SII, 427), being a treatise on moral regulations in society and the market. According to a note on f.la it was translated (tārihh-i terceme) in 969/1561-2.

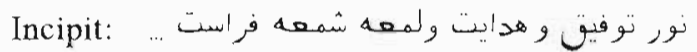
ff. [81] (original foliation 1-14) ; 190 x 130/155 x 80 mm.; 21 lin. - Laid European paper; watermark: paschal lamb; trefoil PP. - Clear neshĩ hand. - Simple golden headpiece; golden double-line rule-borders. - Catchwords. - Text rubricated (overlinings, titles, keywords, quotations). - Side-heads in red. - Decorated paper-covered-boards; no flap.

Anonymous and undated copy (12/18th cent.). Clean copy.

Ref.: OM, III, p. 85.

$$
44 \text { (ISL 61) }
$$

Terceme-i Risāle-i Kudsĩye
Translation and commentary by 'Abdullāh Şalăhī 'Uşşākị (d. 1197/1782-3) on an anonymous al-Risälah al-Qudsĩyah, completed in 1180/1766-7 (chronosticon: zafer), containing sayings (kalimāt) of Bahă al-Din Muhammad Naqshaband al-Bukhārn (d. 791/1389), known as Muhammad Părsā (GAL, SII, 282).

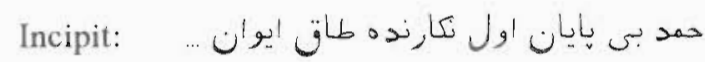

ff. [50] (last folio blank); $197 \times 130 / 135 \times 80$ mm.; 15 lin. - Laid, light brown tinted paper; watermarks: 1. coat of arms (lion rampant), 2. crown and grapes, 3. eagle displayed FG. Elegant neshī hand (Fig. 2). - Illuminated headpiece; single-line red rule-borders. - Text rubricated (overlinings). - Side-heads (matlab). Red leather binding with flap and gilt borders and center-pieces.

Anonymous and undated copy (early $12 / 18^{\text {th }}$ cent.). - Some marginal glosses. - Ownership statement (istashabahu) (f. la): Sharīf Ahmad Muhỹi al-Dìn al-Qādirī, resident (sâkīn) in Tubkhānah (Tophāne), Istanbul at Takiyah Qàdirikhānah (Tekye-i Kãdirihāne) with a seal, dated 1286/ 1869-70: Post-nişin-i. Ķădirihuāne, 1286.

Ref. OM, I, p. 105; EHB, IV, p. 1477.

45 (ISL, 130)

\section{Tezkiret ül-evliya"}

Author: Farīd al-Dín Muhammad al-Atta ar (d. 637/1239-40). - Translation of the Persian dictionary of sufi personalities Tazkirat al-awliyà ' by Hüseyin Enīsī (not mentioned in PFA).

Incipit: الحمد لله رب العالمين والصلوه و السدلام ...

ff. [232]; $227 \times 135 / 140 \times 80$ mm.; 15 lin. Laid European paper; watermarks: 1. three hats, 2. three crescents $\mathrm{A}$. - Neshì hand changing in some places to Ottoman ta'lik.- Text rubricated. - Side-heads (matlab). - Brown paper-covered boards; flap missing.

Copied by (ladá !) Muhammad, known as Jawushzàdah (Çavuşzāde), the Qạdī of the district (bi-qaḍa '/każà') of Qarahyaqah 
(Karayaka) in Safar $1143 / 1730$ (f. 232a). Several seals (some erased) on f. Ia and $231 \mathrm{~b}-$ 232a.

$$
46 \text { (ISL, 105) }
$$

Velāyetnāmè-i Hăcım Sultānn

Author: [Uzun Firdevsi, known as Firdevsi-i Tavil]: - At head of title:

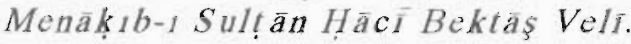

ff. $[54] ; 210 \times 140 / 150 \times 80 \mathrm{~mm} ; 13$ lin. - Buff wove paper; no watermark. - Elegant rik'a hand (Fig. 7). - Catchwords. - Text rubricated. Western style half-leather binding.

Anonymous and undated copy (late $13 / 19^{\text {th }}$ or early $20^{\text {th }}$ cent.). - Former owner: Vakf-1 Dergäh-i Karaağac.

Ref:: TA, XVIII., p. 284; VH.

$$
47 \text { (ISL 118) }
$$

Velayetnāme-i ... Haci Bektās Velī elHorāsāni

Anonymous hagiography of Hacr. Bektaş.

pp. [8] 458; $220 \times 160 / 170 \times 90 \mathrm{~mm}$; 19 lin. Wove and laid (pp. 370-458) European paper; watermarks (laid paper): 1. eagle displayed TSG, 2. coat of arms (lion rampant). - Rik'a kurmasi hand. - Catchwords. - Text rubricated. Orange paper covered boards; flap missing.

Copied by (harrarahu) Husayn Tawfiq, son of "Abd Alläh Baghdādī, Pust-nishīn (Post-nişin) of Q as a bat Mustafá Bāshã (Kașaba-yı Muștafá Paşa), Dhū al-Hijjah 1270/1854, - Main text preceded by table of contents. - Marginal glosses in pencil.

Ref.: TH(4), 151; EHB, V, p. 2015.

48 (ISL 170)

\section{Zeyl-i takvîm üt-tevārih}

Author: Sem'dānizäde Findikli Süleymān Efendi (d. 1193/1779). - Short version of his Mür ${ }^{*} \bar{I}$ üt-tevarih .

ff. 139 (European figures) ; $220 \times 145 / 180 \times$ $130 \mathrm{~mm}$; $23 \mathrm{lin}$ - Modern wove ruled paper. Neat Ottoman rik'a hand. - Anopistograph. Text rubricated. - European style black quarter leather binding.

Copied in September 1929 by 'Oşmān Ferid Bey (f. 139a). - Corrected (tashịh) copy (f. 139a).

Ref.: SF.

\section{Notes}

1 Gacek, Adam. Arabic manuscripts in the libraries of McGill University. Union catalogue. Montreal, McGilll University Libraries, 1991 (Fontanus Monograph Series I); idem. "The diploma of the Egyptian calligrapher Hasan alRushdi", Manuscripts of the Middle East, 4 (1989): 44-55; idem. "Arabic calligraphy and the "Herbal' of al-Ghâfiqî: a survey of Arabic manuscripts at Mct.jill University", Fontanus, 2
(1989): 37-53; idem. "Early Qu'ranic fragments", Fontanus, 3 (1990): 45-64; idemı. "A collection of Qur'anic codices", Fontanus, 4 (1991): 35-53.

2 Bimbaum, Eleazar. "The transliteration of Ottoman Turkish for library and general purposes", Journal of the American Oriental Society, 87 (1967): 122-156. 


\section{Concordance}

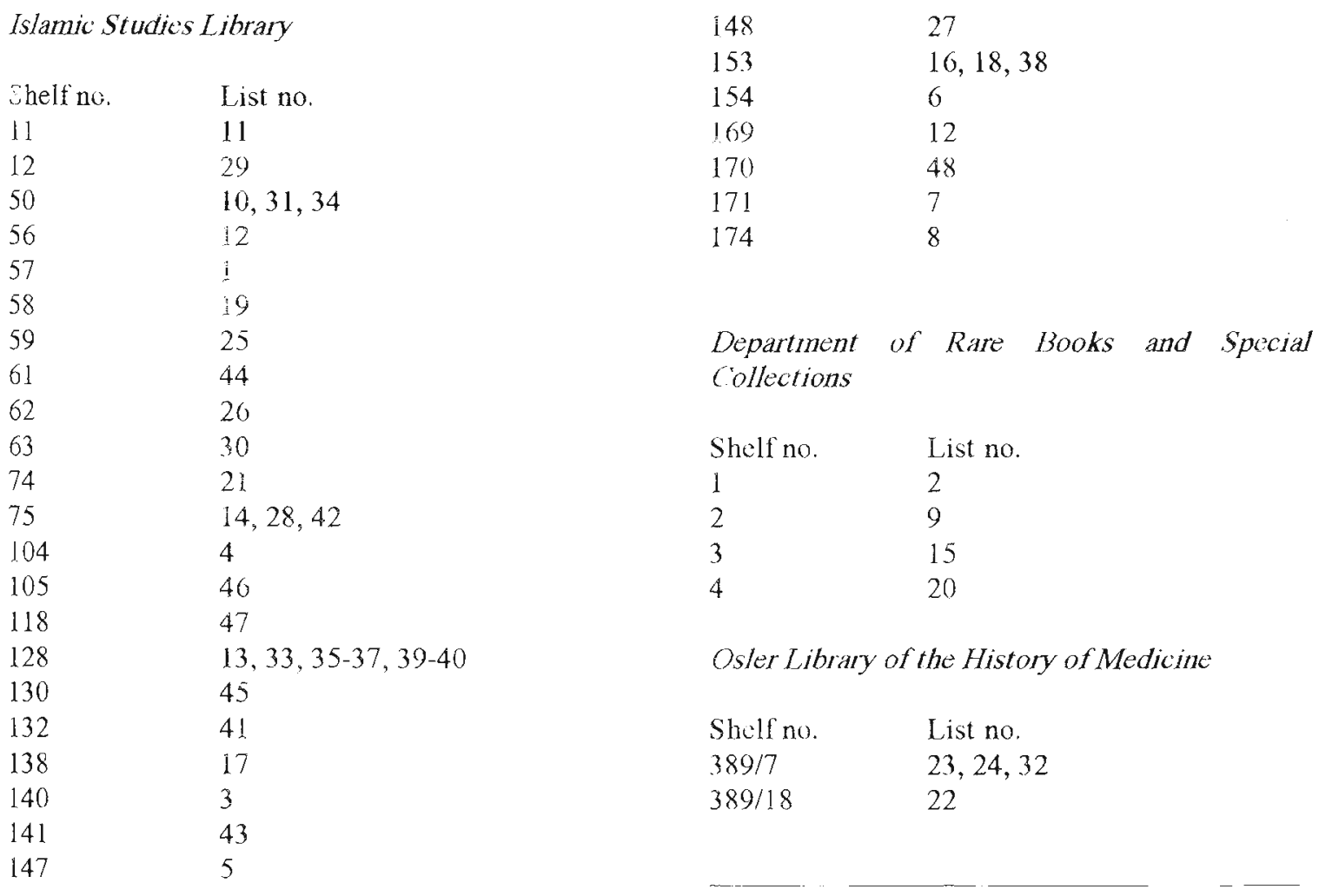



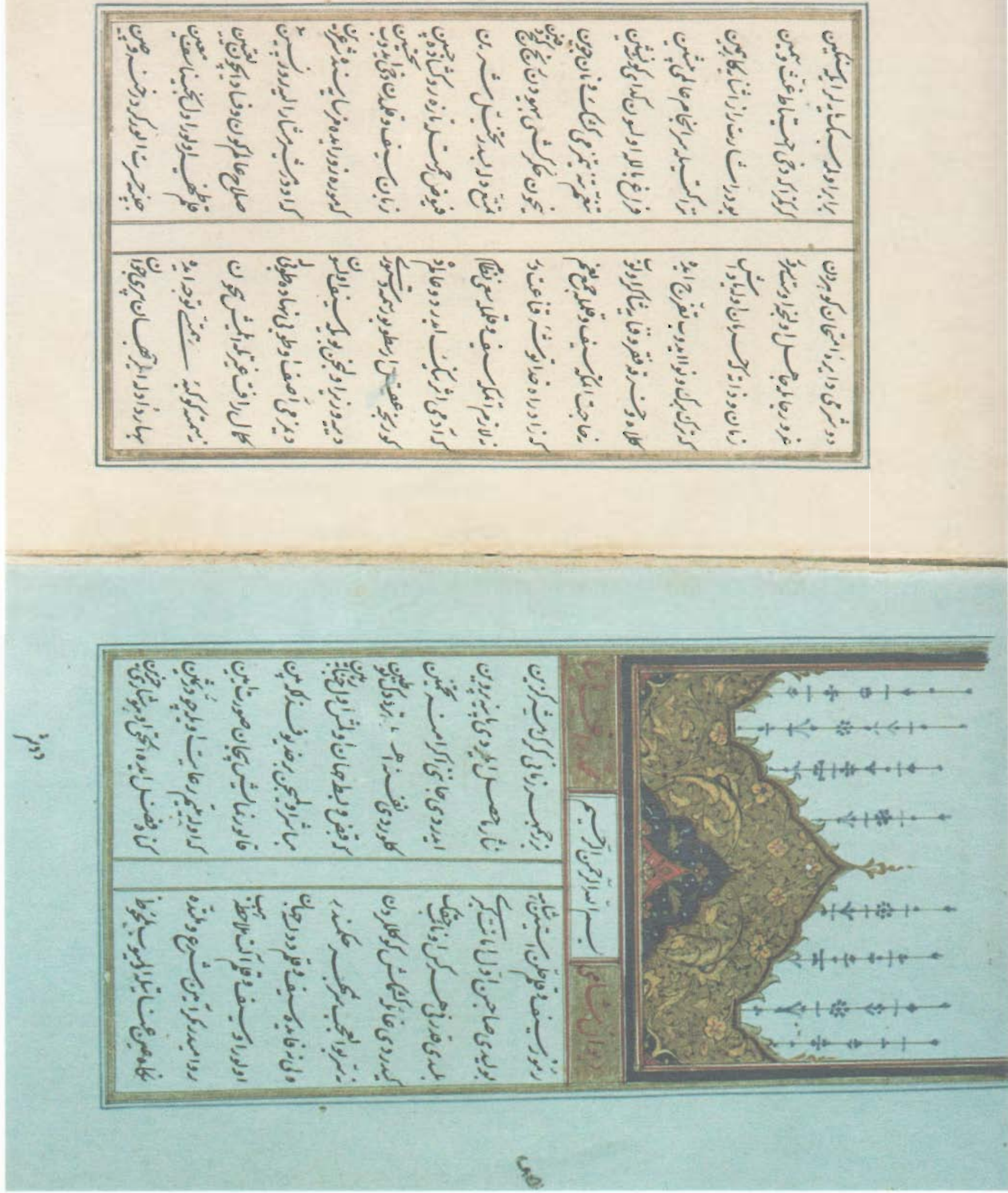


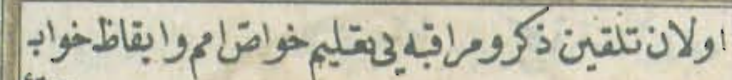

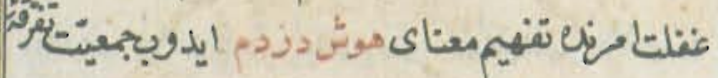
एक ㄱ. 4. ( पर ज "दs (4) जu (1) Es

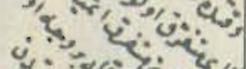

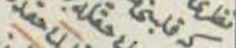
$4,4, x_{0}, x_{0}$ \& 5.48

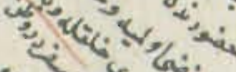
स है है

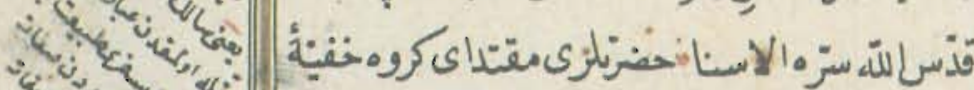

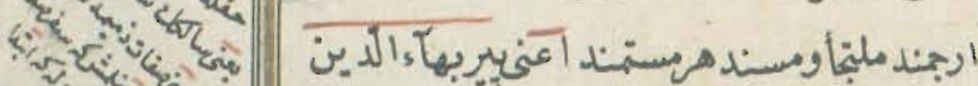

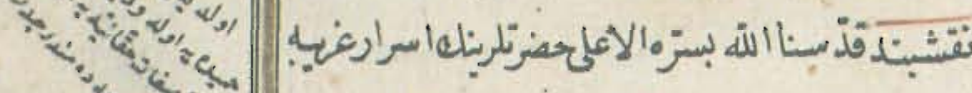

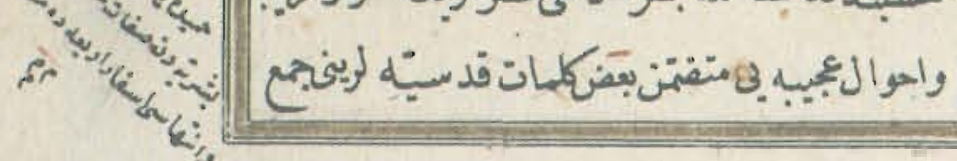

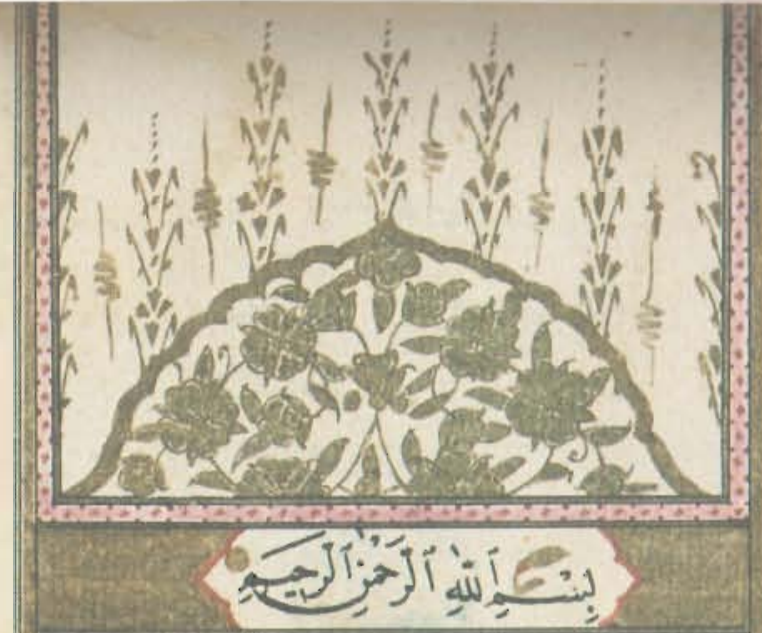

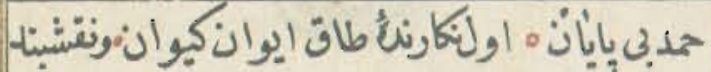

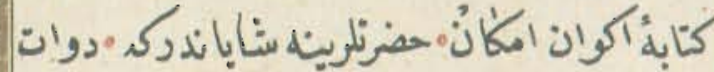

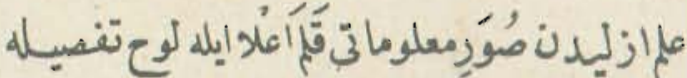

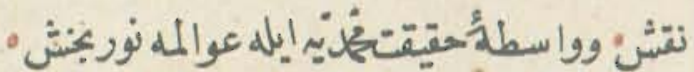

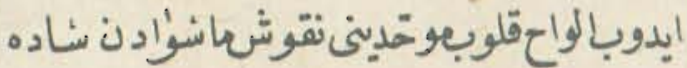

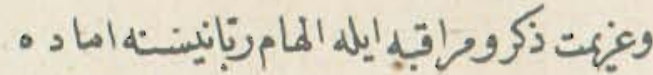

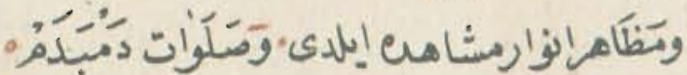

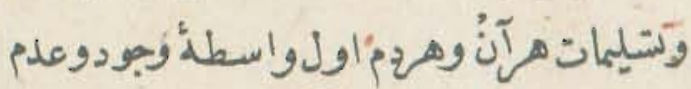

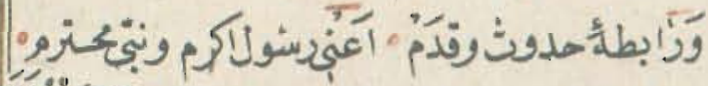

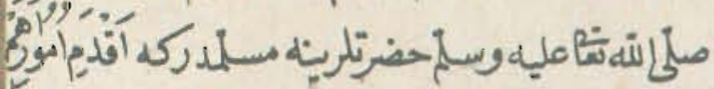




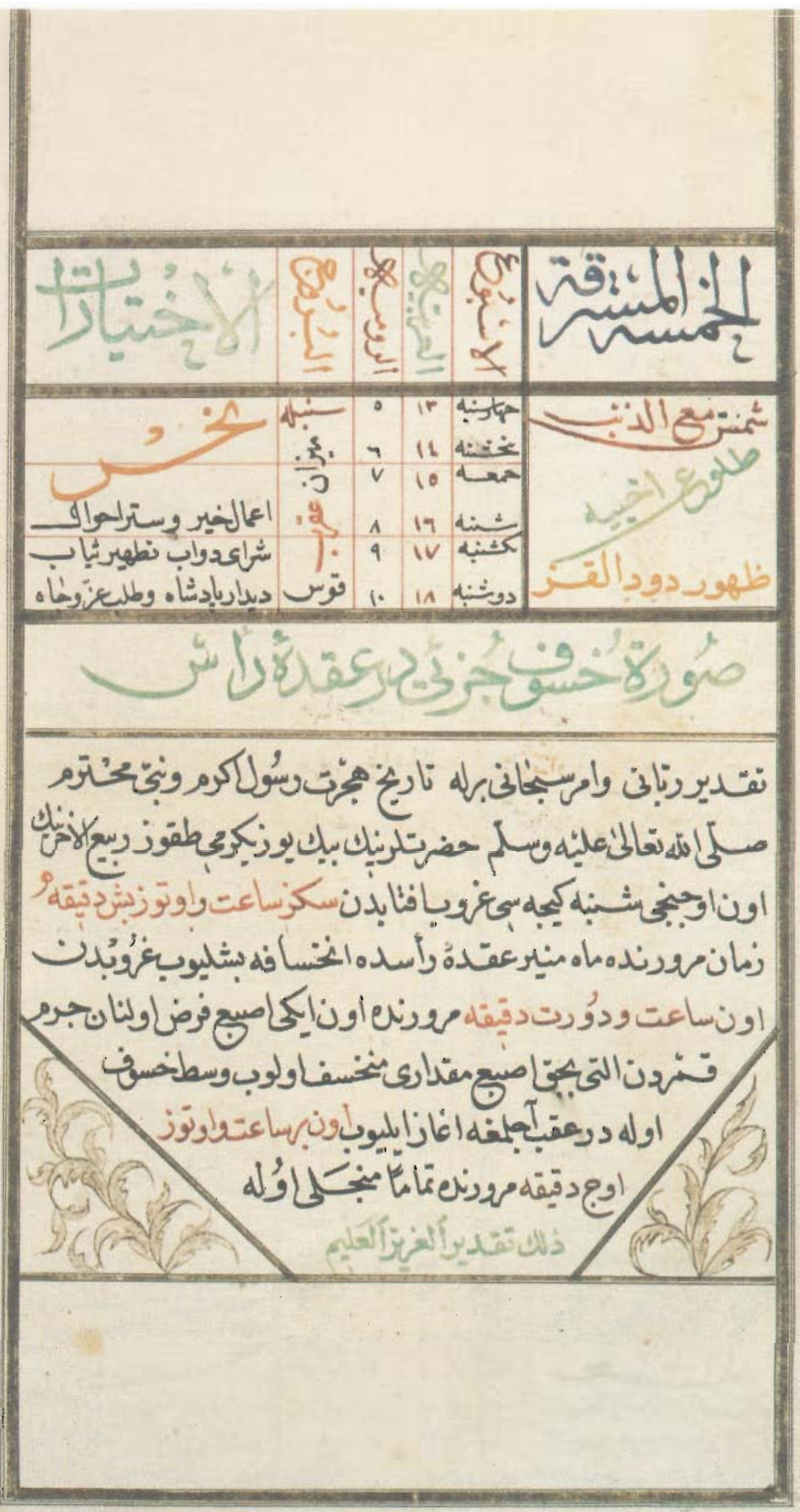

Figure 3. (No. 2, f. 8b) 


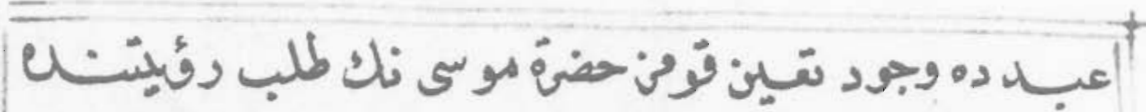

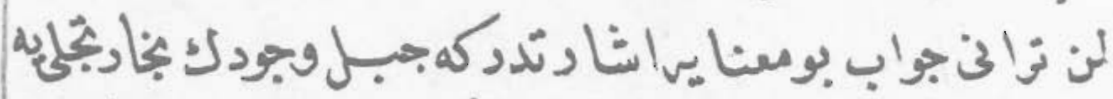

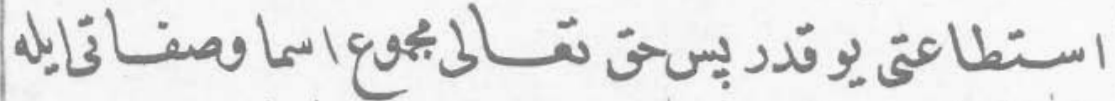

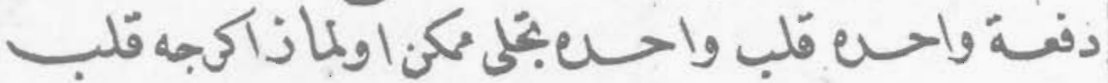

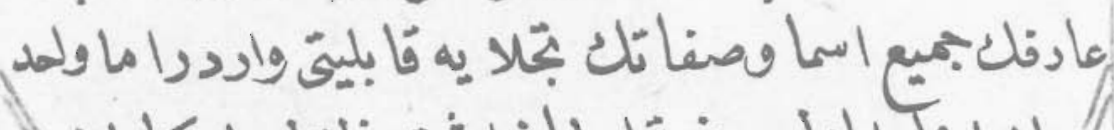

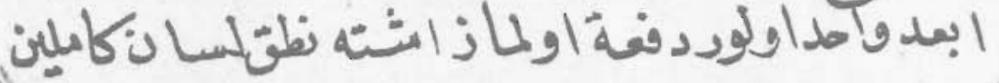

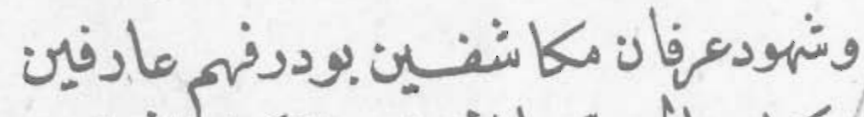

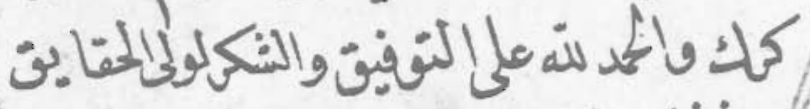

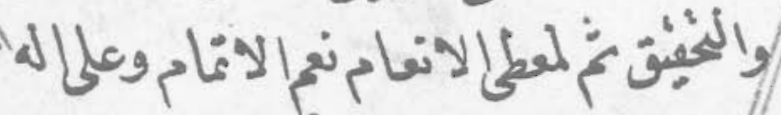

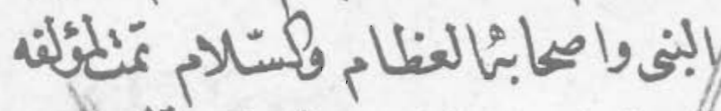

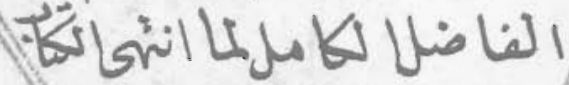

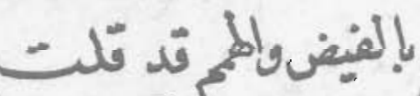

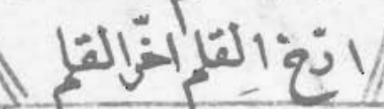

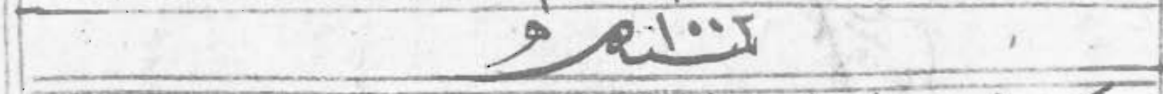

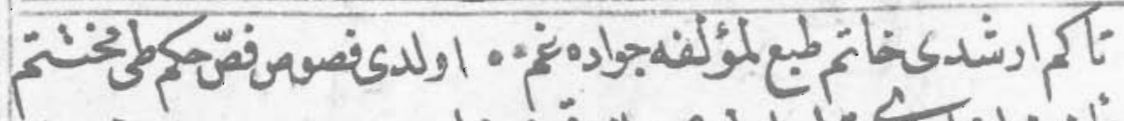

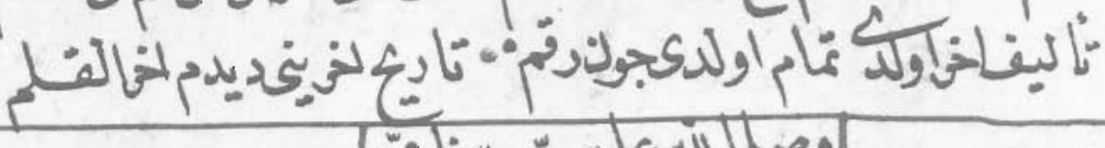

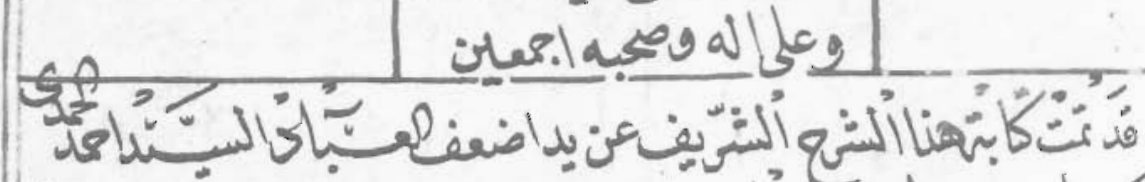

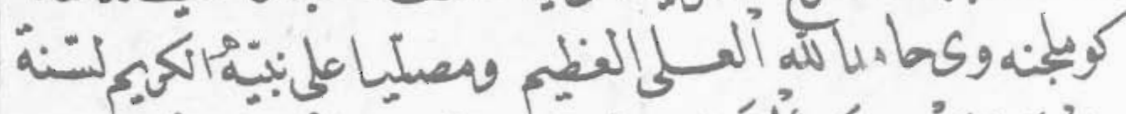

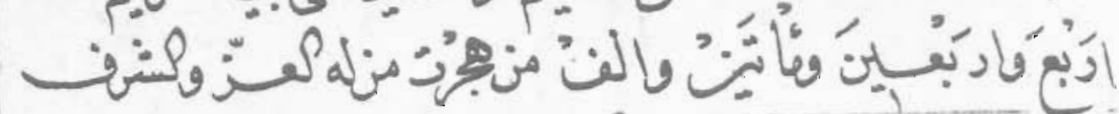

Figure 4. (No. 19, f. 344a) 


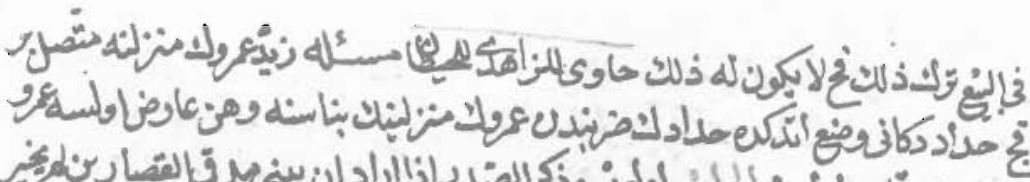

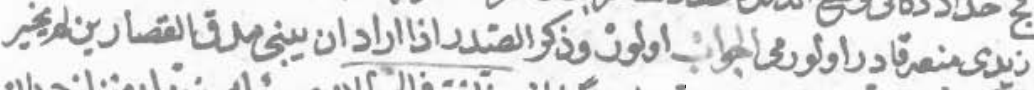

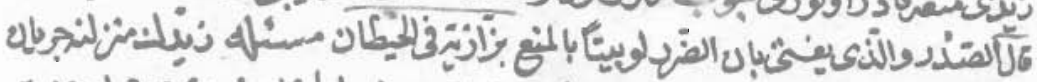

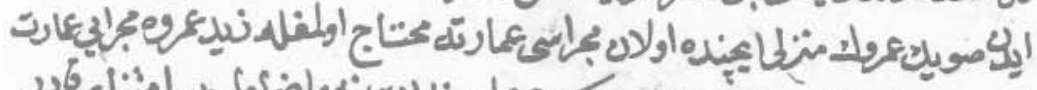

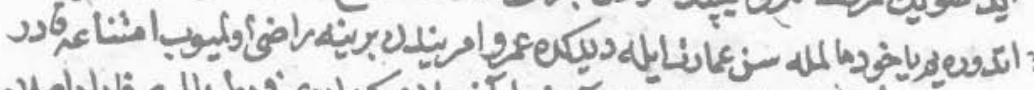

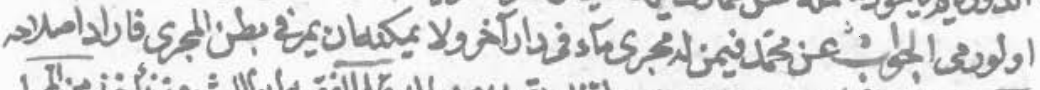

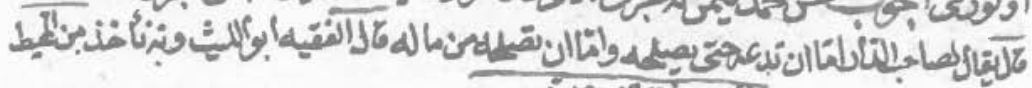

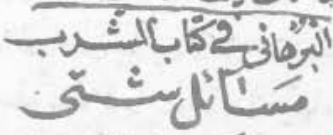

s

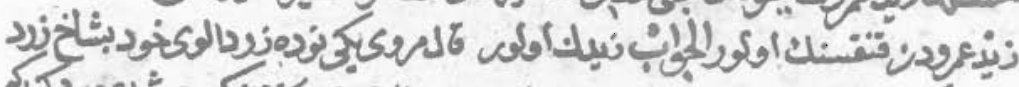

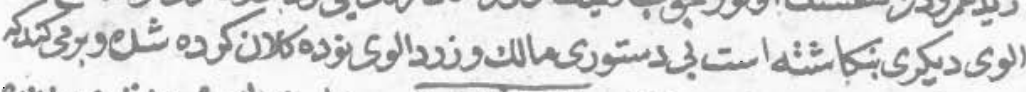

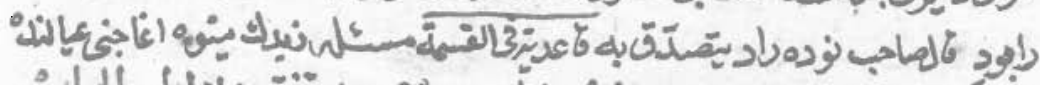

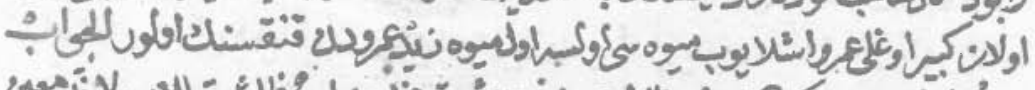

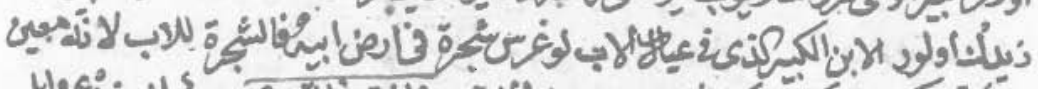

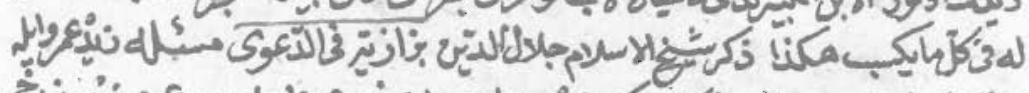

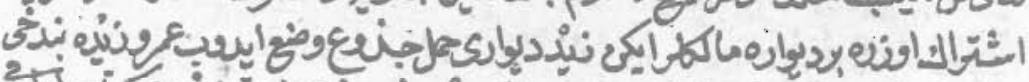

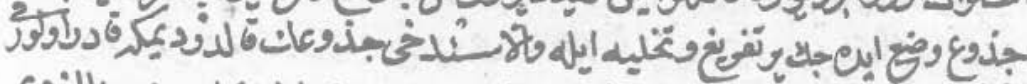

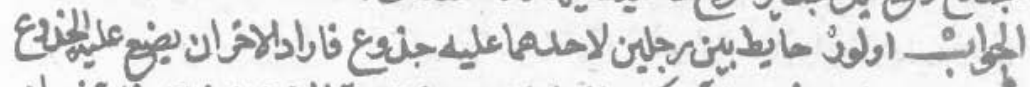

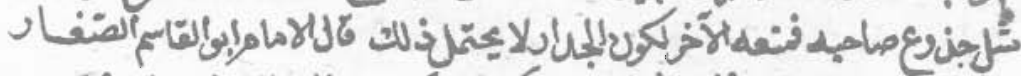

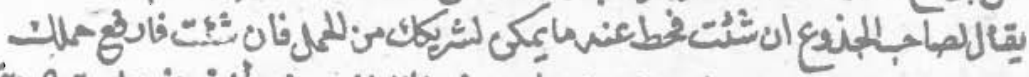

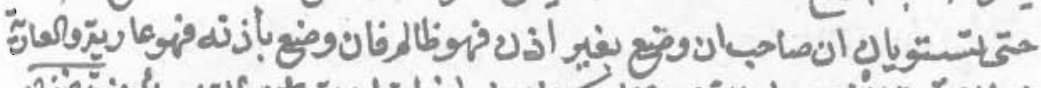

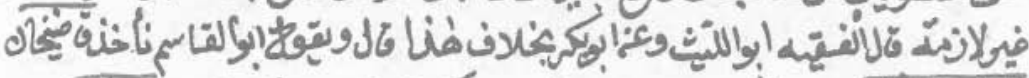

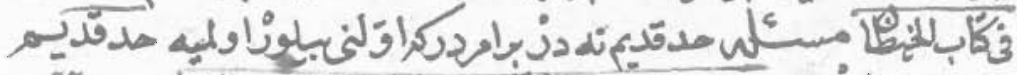

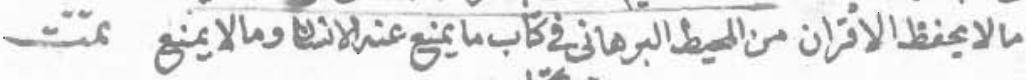
ب: 


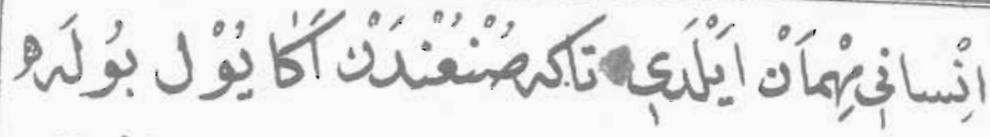

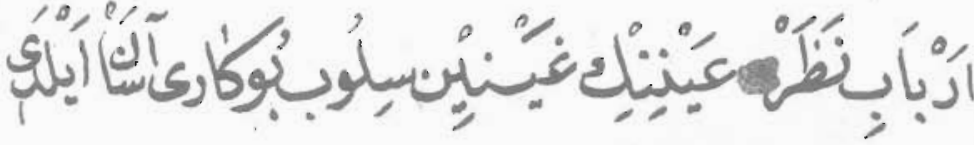

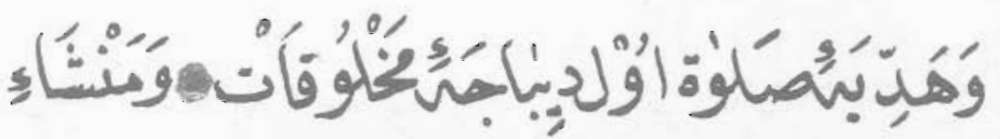

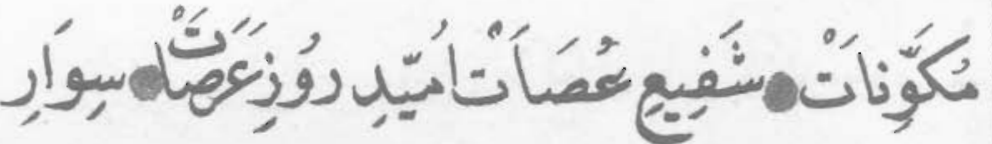

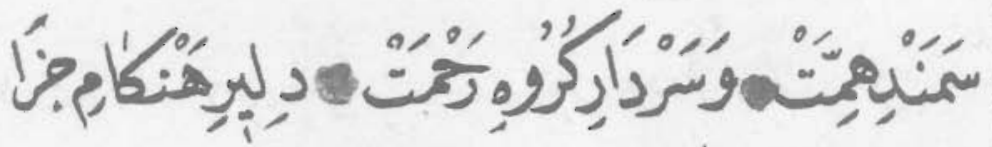

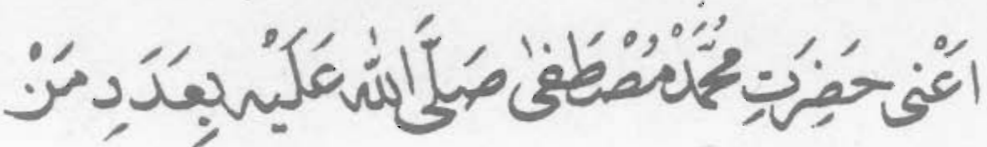

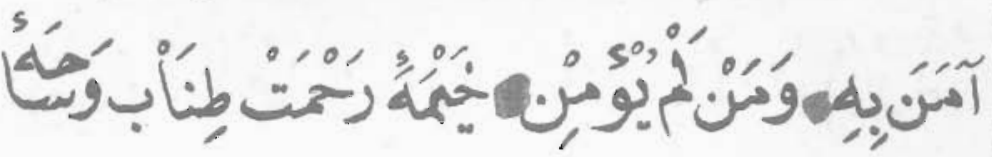

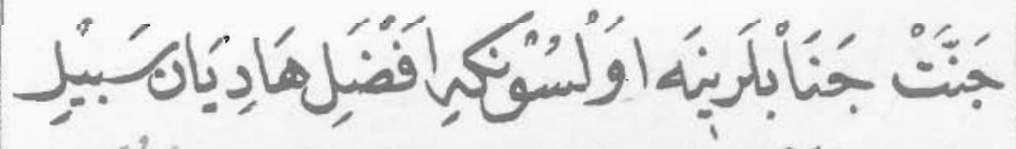

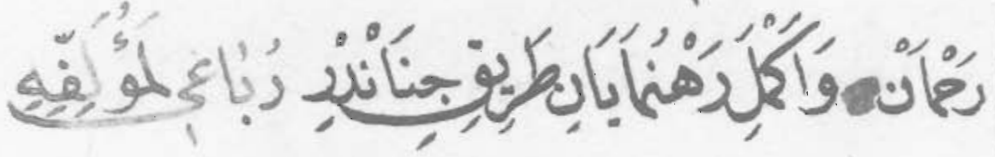

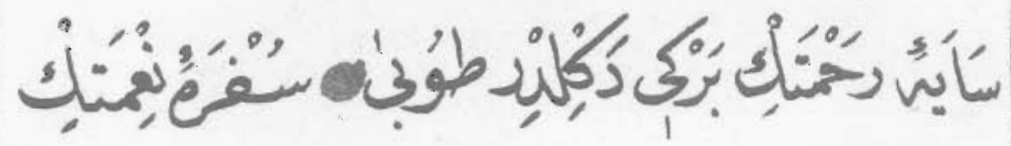

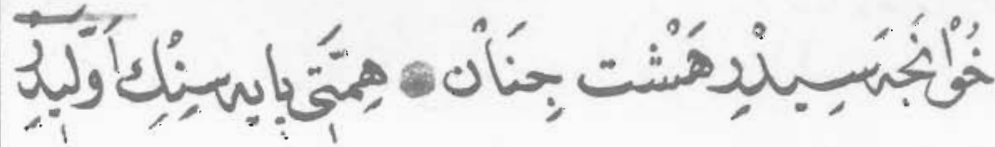

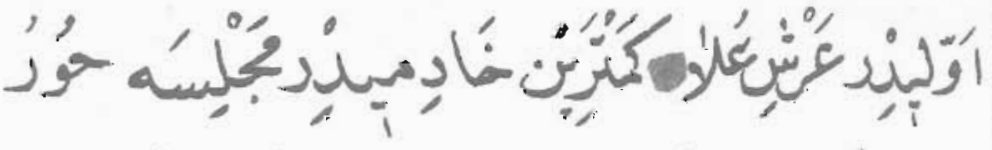

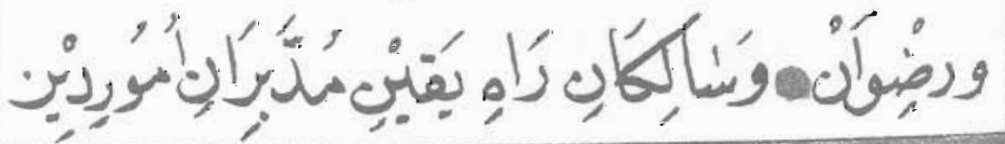

Figure 6. (No. 26, f. 2a) 


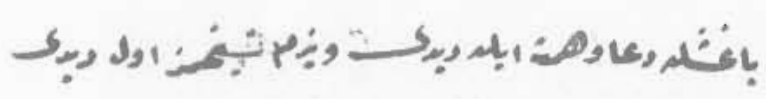

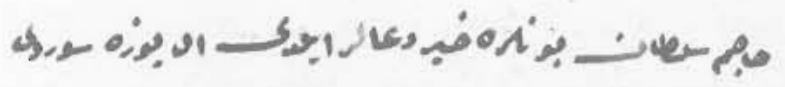

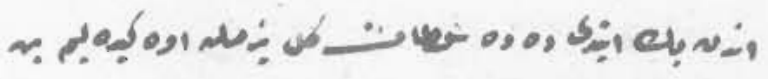

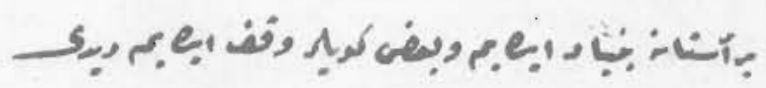

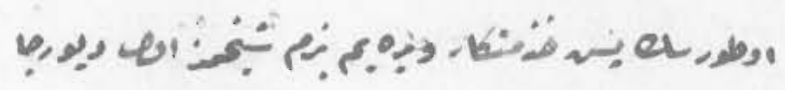

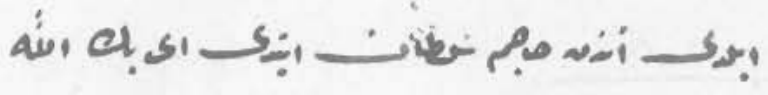

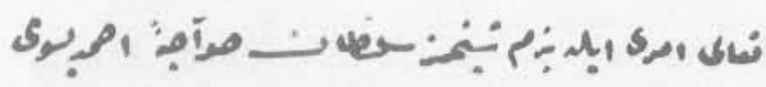

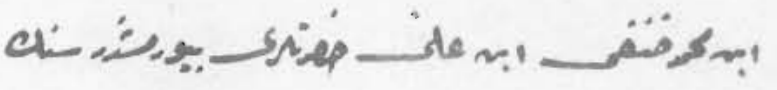

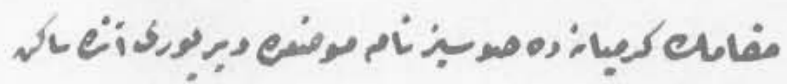

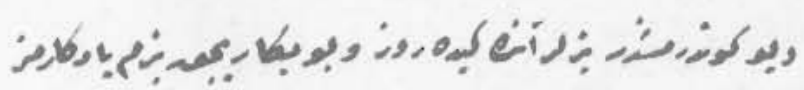

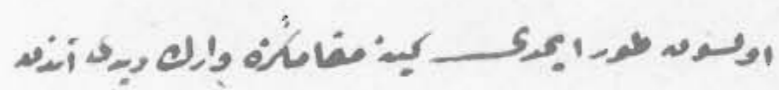

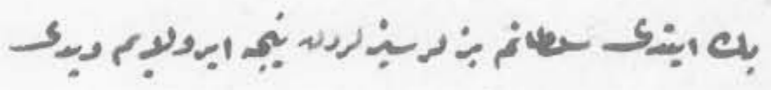

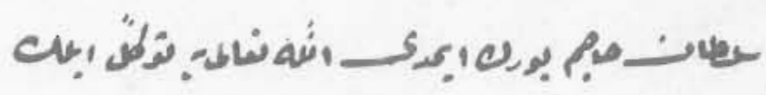

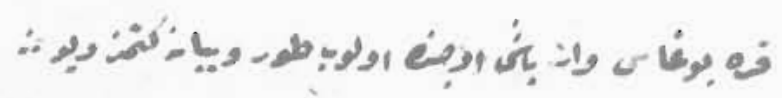

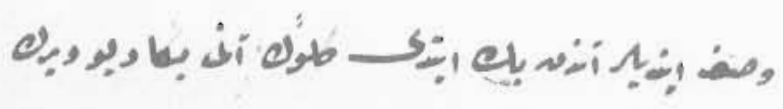

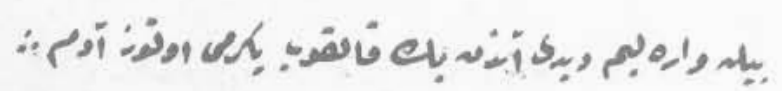

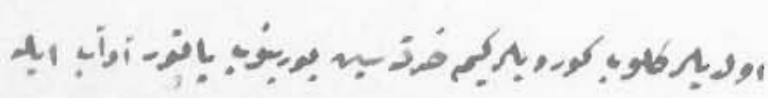

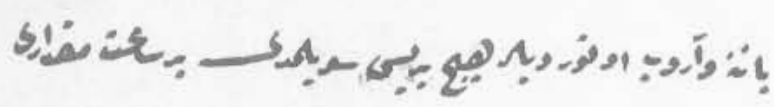

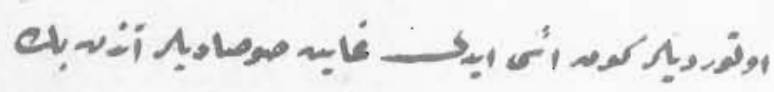

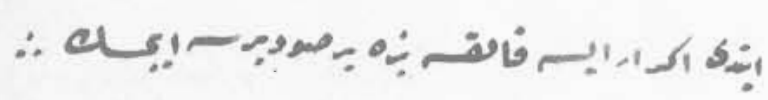

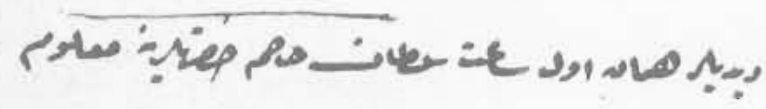

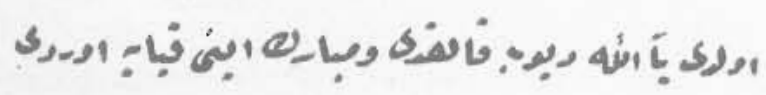

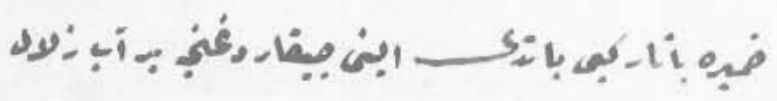
!

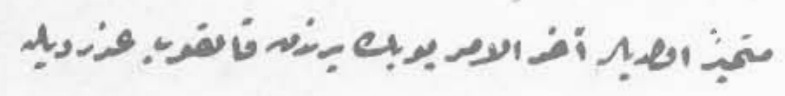

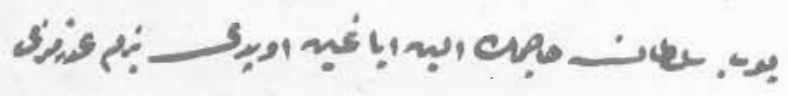
V.ức

Figure 7. (No, 46, f, 20b-21a) 


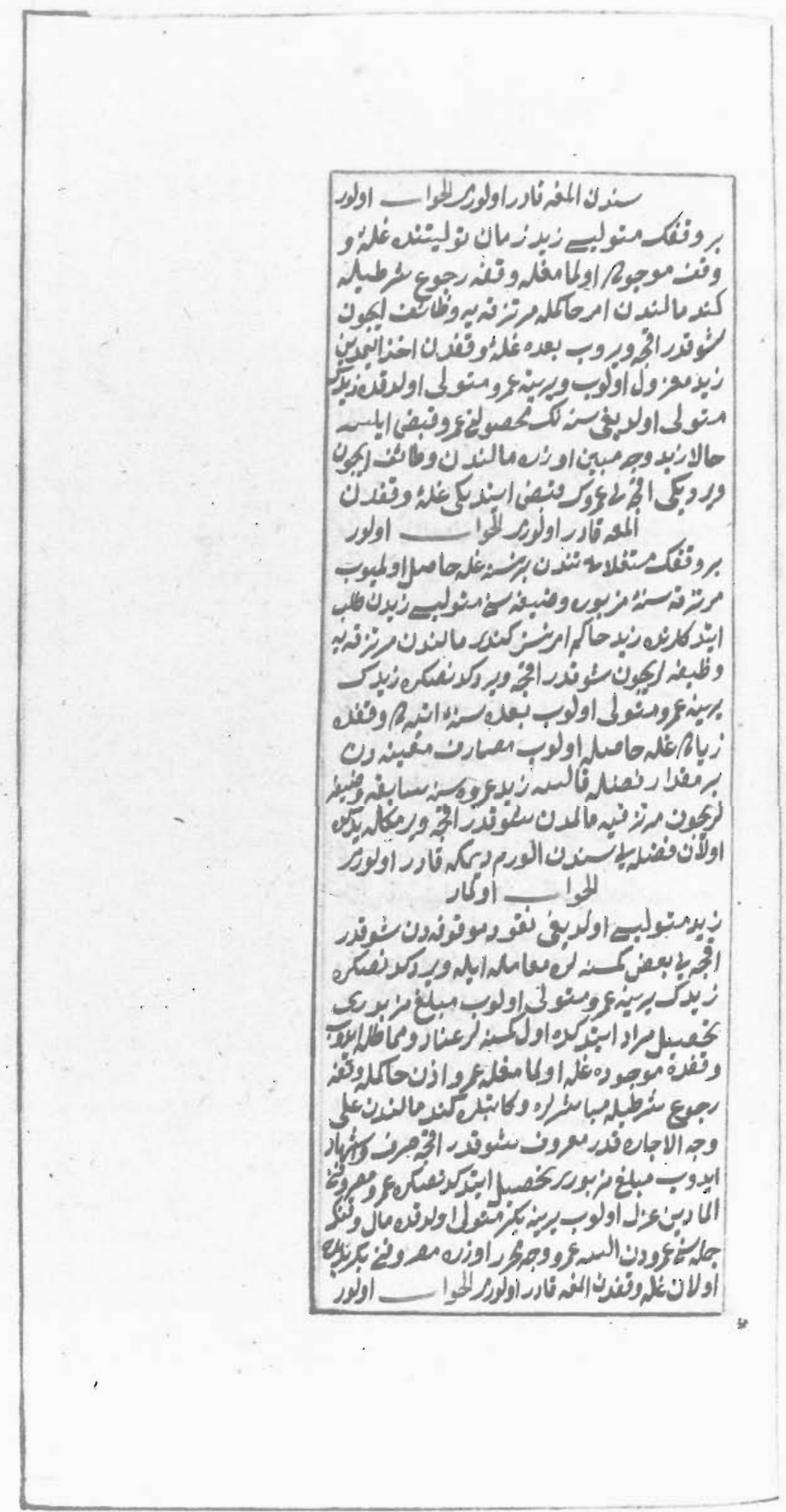

Figure 8. (No. 11, 1. 96a) 